\title{
Dixmier's Problem 6 for the Weyl Algebra (the Generic Type Problem)
}

\author{
V. V. Bavula
}

\begin{abstract}
In the fundamental paper [1], J. Dixmier posed six problems for the Weyl algebra $A_{1}$ over a field $K$ of characteristic zero. Problem 3 was solved by Joseph and Stein [15] (using results of McConnel and Robson [20]), problem 5 was solved by the author in [7. Using a (difficult) polarization theorem for the Weyl algebra $A_{1}$ Joseph 15 solved problem 6. Problems 1, 2, and 4 are still open. Note that these problems make sense for non-commutative algebras of Gelfand-Kirillov $<3$, and some of them (after minor modifications) make sense for an arbitrary noncommutative algebra.

In this paper a short proof is given to Dixmier's problem 6 for many noncommutative algebras $A$ of Gelfand-Kirillov $<3$ (a typical example is the ring of differential operators $\mathcal{D}(X)$ on a smooth irreducible algebraic curve $X$ ). An affirmative answer to this problem leads to clarification of the structure of maximal commutative subalgebras of these algebras $A$ (a typical example of the algebra $A$ is any noncommutative subalgebra of Gelfand-Kirillov $<3$ of the division ring $Q(\mathcal{D}(X))$ for the algebra $\mathcal{D}(X)$ ), and the result is rather surprising: for a given maximal commutative subalgebra $C$ of the algebra $A$, (almost) all non-central elements of it have the same type, more precisely, have exactly one of the following types: (i) strongly nilpotent, (ii) weakly nilpotent, (iii) generic, (iv) generic except for a subset $K^{*} a+Z(A)$ of strongly semi-simple elements, (iv) generic except for a subset $K^{*} a+Z(A)$ of weakly semi-simple elements, where $K^{*}:=K \backslash\{0\}$ and $Z(A)$ is the centre of the algebra $A$.

For an arbitrary algebra $A$, Dixmier's problem 6 is essentially a question: whether an inner derivation of the algebra $A$ of the type ad $f(a), a \in A, f(t) \in K[t]$, $\operatorname{deg}_{t}(f(t))>1$, has a nonzero eigenvalue. We prove that the answer is negative for many classes of algebras (eg, rings of differential operators $\mathcal{D}(Y)$ on smooth irreducible algebraic varieties, all prime factor algebras of the universal enveloping algebra $U(\mathcal{G})$ of a completely solvable algebraic Lie algebra $\mathcal{G}$ ).
\end{abstract}

\section{Introduction}

In this paper, $K$ is a field of characteristic zero and $\bar{K}$ is its algebraic closure.

The (first) Weyl algebra $A_{1}$ is an associative algebra generated over the field $K$ by elements $x$ and $\partial$ that satisfy the defining relation $\partial x-x \partial=1$. The Weyl algebra $A_{1}$ is a central, simple, Noetherian domain of Gelfand-Kirillov dimension 2 which is canonically 
isomorphic to the ring of differential operators $K[x]\left[\frac{d}{d x}\right]\left(x \leftrightarrow x, \partial \leftrightarrow \frac{d}{d x}\right)$ with coefficients from the polynomial algebra $K[x]$. The $n$ 'th Weyl algebra $A_{n}$ is the tensor product $A_{1} \otimes$ $\cdots \otimes A_{1}$ of $n$ copies of the first Weyl algebra.

In the seminal paper [11, Dixmier initiated a systematic study of the structure of the first Weyl algebra. At the end of this paper he posed six problems (questions). Problem 3 has been solved by Joseph and Stein [15] (using results of McConnel and Robson [20]), problem 5 has been solved by the author in [7. Using a (difficult) polarization theorem for the Weyl algebra $A_{1}$ Joseph [15] solved problem 6. Problems 1, 2, and 4 are still open. Dixmier's problem 1: whether an algebra endomorphism of the first Weyl algebra is an algebra automorphism? He writes in the paper on page 242 that " $A$. A. Kirillov informed me that the Moscow school also considered this problem." A positive answer to a similar problem but for the $n$ 'th Weyl algebra implies the Jacobian Conjecture (see the paper of Bass, Connel and Wright [3] for details, and [6] on a progress on these two problems).

Let $A$ be an algebra over the field $K$, and let $\operatorname{Der}_{K}(A)$ be the set of all $K$-derivations of the algebra $A$. We can identify the algebra $A$ with its image in the algebra $\bar{A}:=\bar{K} \otimes_{K} A$ via

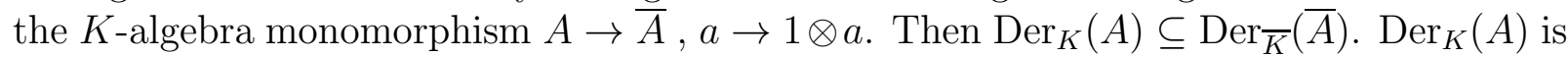
a Lie algebra with the bracket given by the commutator of derivations $([\delta, \partial]:=\delta \partial-\partial \delta)$. For each derivation $\delta \in \operatorname{Der}_{K}(A)$ of the algebra $A$ one can attach four subalgebras of $A$ : the kernel $C(\delta):=\operatorname{ker} \delta$; the (N-filtered) nil-algebra $N(\delta):=\bigcup_{i>0} N(\delta, i)$ where $N(\delta, i):=$ $\operatorname{ker} \delta^{i+1}$; the eigenvalue algebra $D(\delta):=A \cap D(\delta, \bar{A})$ for $\delta$ where the subalgebra $D(\delta, \bar{A})$ of $\bar{A}$ is the direct sum

$$
\bigoplus_{\lambda \in \operatorname{Ev}(\delta, \bar{A})} D(\delta, \lambda, \bar{A})
$$

of the eigenspaces $D(\delta, \lambda, \bar{A}):=\{a \in \bar{A} \mid \delta(a)=\lambda a\}$ belonging to the eigenvalue $\lambda \in$ $\bar{K}, \operatorname{Ev}(\delta, \bar{A})$ is the set of all eigenvalues for $\delta$ in $\bar{A}$; the torsion algebra $F(\delta):=\{a \in$ $\left.A \mid \operatorname{dim}_{K} K[\delta] a<\infty\right\}$ for $\delta$. Clearly, $C(\delta)=N(\delta) \cap D(\delta), N(\delta) \subseteq F(\delta)$, and $D(\delta) \subseteq F(\delta)$.

The set $\operatorname{Der}_{K}(A) \backslash\{0\}$ is a disjoint union of its eleven subsets

$$
\operatorname{Der}_{K}(A) \backslash\{0\}=\bigcup_{i=1}^{11} \widetilde{\Delta}_{i}(A)
$$

where

1. $\widetilde{\Delta}_{1}(A)=\{\delta: N(\delta)=A, D(\delta)=C(\delta)\}$, a set of strongly nilpotent derivations.

2. $\widetilde{\Delta}_{2}(A)=\{\delta: C(\delta) \neq N(\delta) \neq A, D(\delta)=C(\delta)\}$, a set of weakly nilpotent derivations.

3. $\widetilde{\Delta}_{3}(A)=\{\delta: N(\delta)=C(\delta), D(\delta)=A\}$, a set of strongly semi-simple derivations.

4. $\widetilde{\Delta}_{4}(A)=\{\delta: N(\delta)=C(\delta), C(\delta) \neq D(\delta)=F(\delta) \neq A\}$, a set of weakly semi-simple derivations.

5. $\widetilde{\Delta}_{5}(A)=\{\delta: N(\delta)=D(\delta)=C(\delta)\}$, a set of generic derivations. 
6. $\widetilde{\Delta}_{6}(A)=\{\delta: N(\delta)=C(\delta) \neq D(\delta) \neq F(\delta)=A\}$, a set of strongly jordan derivations.

7. $\widetilde{\Delta}_{7}(A)=\{\delta: N(\delta)=C(\delta) \neq D(\delta) \neq F(\delta) \neq A\}$, a set of weakly jordan derivations.

8. $\widetilde{\Delta}_{8}(A)=\{\delta: N(\delta) \neq C(\delta) \neq D(\delta), N(\delta)+D(\delta) \neq F(\delta)=A\}$, a set of strongly nil-jordan derivations.

9. $\widetilde{\Delta}_{9}(A)=\{\delta: N(\delta) \neq C(\delta) \neq D(\delta), N(\delta)+D(\delta) \neq F(\delta) \neq A\}$, a set of weakly nil-jordan derivations.

10. $\widetilde{\Delta}_{10}(A)=\{\delta: N(\delta) \neq C(\delta) \neq D(\delta), N(\delta)+D(\delta)=A\}$, a set of strongly nil-semisimple derivations.

11. $\widetilde{\Delta}_{11}(A)=\{\delta: \quad N(\delta) \neq C(\delta) \neq D(\delta), N(\delta)+D(\delta)=F(\delta) \neq A\}$, a set of weakly nil-semi-simple derivations.

For each element $a \in A$, one can attach, so-called, the inner derivation ad $a$ of the algebra $A$ by the rule ad $a(b):=[a, b]=a b-b a$, and then four subalgebras of $A$ : the centralizer $C(a):=C(\operatorname{ad} a)=\{b \in A \mid a b=b a\}$ of the element $a$ in $A, N(a):=N(\operatorname{ad} a), D(a):=$ $D(\operatorname{ad} a)$, and $F(a):=F(\operatorname{ad} a)$. The algebra $A$ is a Lie algebra where the bracket is the commutator of elements. The map ad : $A \rightarrow \operatorname{Der}_{K}(A), a \rightarrow$ ad $a$, is a Lie algebra homomorphism with kernel $Z(A)$, the centre of the algebra $A$.

The set $A \backslash Z(A)$ is a disjoint union of its eleven subsets

$$
A \backslash Z(A)=\bigcup_{i=1}^{11} \Delta_{i}(A) \text { where } \Delta_{i}(A):=\left\{a \in A \backslash Z(A) \mid \operatorname{ad} a \in \widetilde{\Delta}_{i}(A)\right\} .
$$

Elements of the sets $\Delta_{1}(A), \ldots, \Delta_{11}(A)$ are called respectively strongly nilpotent, ..., weakly nil-semi-simple. The sets $\Delta_{i}(A)$ are invariant under the action of the group $\operatorname{Aut}_{K}(A)$ of all $K$-algebra automorphisms of $A$.

Definition. We say that an algebra $A$ admits the Dixmier partition if

$$
A \backslash Z(A)=\Delta_{1}(A) \cup \Delta_{2}(A) \cup \Delta_{3}(A) \cup \Delta_{4}(A) \cup \Delta_{5}(A) .
$$

Equivalently, an algebra $A$ admits the Dixmier partition if and only if, for each $a \in A \backslash Z(A)$, either $F(a)=N(a)$ or $F(a)=D(a)$ (the nilpotent-semi-simple alternative).

Dixmier [11] proved that the first Weyl algebra $A_{1}$ satisfies this property. One can modify arguments of Dixmier and prove that each non-commutative algebra $A$ of GelfandKirillov dimension $<3$ admits the Dixmier partition provided $\bar{A}$ is a domain (Corollary 3.3).

Dixmier's problem 6, 11]: If $a \in \Delta_{5}\left(A_{1}\right)$ then $K[a] \backslash K \subseteq \Delta_{5}\left(A_{1}\right)$ ?

The type (properties) of an element $a \in A_{1}$ is determined by the type of the corresponding inner derivation ad $a$. So, Dixmier's problem 6 is a question about properties of inner derivations. Dixmier proved in his paper [1], 10.3, that, for $i=1,2$ and any non-scalar polynomial $f(t), a \in \Delta_{i}\left(A_{1}\right) \Leftrightarrow f(a) \in \Delta_{i}\left(A_{1}\right)$. So, for an arbitrary algebra $A$, Dixmier's problem 6 is essentially a question of 
- whether an inner derivation of the type ad $f(a)$ has a non-zero eigenvalue (where $a \in A, f(t) \in K[t], \operatorname{deg} f(t)>1)$.

The answer is negative for many classes of algebras (rings of differential operators on smooth irreducible algebraic varieties, universal enveloping algebras of Lie algebras) as follows from the next result (Section 2, see Theorem 2.1 for a more general result).

Theorem 1.1 Let $B=\cup_{i \geq 0} B_{i}$ be a filtered algebra over a field $K$ of characteristic zero such that the associated graded algebra gr $B$ is a commutative domain, and let $a \in B$. Then, for an arbitrary polynomial $f(t) \in K[t]$ of degree $>1,0$ is the only eigenvalue of the inner derivation ad $f(a)$ of the algebra $B$ (that is, if $\delta:=\operatorname{ad} f(a) \neq 0$ and $\delta(b)=\lambda b$ for some $\lambda \in K$ and $0 \neq b \in B$ then $\lambda=0)$.

As an immediate consequence of Theorem 1.1 we have the following result (among others of the same sort, Section (2) about nonexistence of nonzero eigenvalue for inner derivations of the type ad $f(a)$.

Theorem 1.2 Let $\mathcal{D}(X)$ be the ring of differential operators on a smooth irreducible affine algebraic variety $X$ over a field $K$ of characteristic zero, and let $a \in \mathcal{D}(X)$. Then, for an arbitrary polynomial $f(t) \in K[t]$ of degree $>1,0$ is the only eigenvalue of the inner derivation ad $f(a)$ of $\mathcal{D}(X)$.

Proof (see Section 2for detail). By the very definition of the ring of differential operators $\mathcal{D}(X)$, the algebra $\mathcal{D}(X)$ has the order filtration $\mathcal{D}(X)=\cup_{i \geq 0} \mathcal{D}(X)_{i}$ (by the total degree of derivations) such that the associated graded algebra gr $\mathcal{D}(X)$ is a commutative domain, [21], 15.4.7 and 15.5.6. So, the result follows from Theorem 1.1.

The same result is true $(i)$ for all prime factor algebras of the universal enveloping algebra $U(\mathcal{G})$ where $\mathcal{G}$ is a completely solvable, algebraic Lie algebra (Corollary 2.5); or $\mathcal{G}$ is a nilpotent Lie algebra (Corollary [2.3); (ii) for all primitive factor algebras of the universal enveloping algebra $U(\mathcal{G})$ where $\mathcal{G}$ is a solvable Lie algebra (Corollary 2.6); (iii) for algebra $\mathcal{A}(V, \delta, \Gamma)$ from [21], Ch. 14, Section 8.

Now we are ready to give a short proof to Dixmier's problem 6 .

Proof (Dixmier's problem 6). We may assume that $K=\bar{K}$ (since $\bar{A}_{1}$ is a domain). Recall that the Weyl algebra $A_{1}$ satisfies the Dixmier partition [11]. The Weyl algebra $A_{1}$ has a filtration by the total degree of the canonical generators:

$$
A_{1}=\bigcup_{n \geq 0} A_{1, n} \text { where } A_{1, n}:=\sum_{i, j \geq 0}\left\{K x^{i} \partial^{j} \mid i+j \leq n\right\} .
$$

The associated graded algebra gr $A_{1}:=\oplus_{n \geq 0} A_{1, n} / A_{1, n-1}$ is a polynomial algebra $K[\bar{x}, \bar{\partial}]$ in two variables $\bar{x}:=x+A_{1,0}$ and $\bar{\partial}:=\partial+A_{1,0}$ where $A_{1,0}=K$ and $A_{1,-1}:=0$.

Let $a \in \Delta_{5}\left(A_{1}\right)$ and $f(t) \in K[t], \operatorname{deg} f(t)>1$. By Theorem 1.1. $f(a) \notin \Delta_{3,4}\left(A_{1}\right)$. By the result of Dixmier [1], 10.3 (mentioned above), $f(a) \notin \Delta_{1,2}\left(A_{1}\right)$. Hence $f(a) \in \Delta_{5}\left(A_{1}\right)$. 
Dixmier's question 6 is the most difficult part in an answer to the question: suppose that the type of an element $a$ is known. What is the type of the element $f(a)$ where $f(t) \in K[t]$, $\operatorname{deg}_{t} f(t)>1$ ? For the Weyl algebra $A_{1}$ the answer is

1. Let $i=1,2,5$. If $a \in \Delta_{i}\left(A_{1}\right)$ then $f(a) \in \Delta_{i}\left(A_{1}\right)$.

2. Let $i=3$, 4. If $a \in \Delta_{i}\left(A_{1}\right)$ then $f(a) \in \Delta_{5}\left(A_{1}\right)$.

Proof. For $i=1,2$, this is the result of Dixmier [11, 10.3. For $i=5$, this is Dixmier's question 6. For $i=3,4, f(a) \notin \Delta_{3,4}\left(A_{1}\right)$ (see the proof to Dixmier's problem 6), and $f(a) \notin \Delta_{1,2}\left(A_{1}\right)$, by [11], 10.3, and so $f(a) \in \Delta_{5}\left(A_{1}\right)$.

Exactly the same result holds for the ring of differential operators $\mathcal{D}(X)$ on a smooth irreducible algebraic curve $X$ (Corollary 7.4), or even in more general situation (Theorem 4.6).

The elements $a$ and $f(a)$ commute. One can ask a similar question but for commuting elements.

Question. Suppose that the type of an element a is known. What are the types of elements commuting with a?

For many classes of algebras an answer is given by the following definition (see Theorem 1.3 and the examples that follow).

Definition. Let $A$ be a noncommutative algebra that admits the Dixmier partition. We say that $A$ satisfies the homogeneous centralizer condition (the $h c c$, for short) if, for each element $a \in A \backslash Z(A)$, all the elements of the set $C(a) \backslash Z(A)$ have exactly one of the following types:

1. strongly nilpotent,

2. weakly nilpotent,

3. generic except for a subset $K^{*} a+Z(A)$ of strongly semi-simple elements for some $a \in \Delta_{3}(A)$ where $K^{*}:=K \backslash\{0\}$,

4. generic except for a subset $K^{*} a+Z(A)$ of weakly semi-simple elements for some $a \in \Delta_{4}(A)$,

5. generic.

The next definition is inspired by the result of Flanders (see the review of Ore of the paper [1] on MathSciNet) and Amitsur [1]: Let a be a non-scalar element of the Weyl algebra $A_{1}$. Then its centralizer $C(a)$ is a commutative subalgebra of $A_{1}$, and a free, finitely generated $K[a]$-module.

Definition. A noncommutative algebra $A$ satisfies the commutative centralizer condition (the $c c c$, for short) if the centralizer of each element of the set $A \backslash Z(A)$ is a commutative algebra.

When this paper was written D. Jordan and T. Lenagan pointed out on a paper of K. R. Goodearl on centralizers [14] where the reader can find numerous examples of algebras that satisfy the commutative centralizer condition. 
Suppose that an algebra $A$ satisfies the commutative centralizer condition. Then, for each element $a \in A \backslash Z(A)$, its centralizer $C(a)$ is a unique maximal commutative subalgebra of $A$ that contains the element $a$, and each maximal commutative subalgebra $C$ of $A$ coincides with the centralizer of every element of $C \backslash Z(A)$ (Corollary 4.1). So, for an algebra that satisfies the $c c c$ the concepts of centralizer and of maximal isotropic subalgebra coincide. For $a, b \in A \backslash Z(A)$, either $C(a)=C(b)$ or otherwise $C(a) \cap C(b)=Z(A)$ (Corollary 4.2), and so every algebra that satisfies the $c c c$ is a union of distinct centralizers (= maximal commutative subalgebras) that meet at $Z(A)$. If, in addition, the algebra $A$ admits the Dixmier partition and satisfies the $h c c$, then this union $A=\cup C(a)$ has a transparent (homogeneous) structure. One of the goals of this paper is to prove that many popular algebras admit the Dixmier partition and satisfy both the homogeneous centralizer condition and the commutative centralizer condition.

The following statement is one of the main results of the paper (Section 41).

Theorem 1.3 Let a $\bar{K}$-algebra $\Gamma$ be a division ring that satisfies the commutative centralizer condition, and let $A$ be a non-commutative $K$-algebra of Gelfand-Kirillov dimension $<3$ over $K$ such that $\bar{A}$ is (isomorphic to) a $\bar{K}$-subalgebra of $\Gamma$. Then $A$ admits the Dixmier partition and satisfies both the homogeneous centralizer condition and the commutative centralizer condition.

Let $\mathcal{D}(X)$ be the ring of differential operators on a smooth irreducible algebraic curve $X$ over $K=\bar{K}$. Then the division ring $Q(\mathcal{D}(X))$ of the algebra $\mathcal{D}(X)$ satisfies the $c c c$ (Corollary [7.2). Let $\mathcal{L}=L\left[\left[X, X^{-1} ; \sigma\right]\right]$ be the skew Laurent series algebra with coefficients from a field $L$ where an automorphism $\operatorname{id}_{L} \neq \sigma \in \operatorname{Aut}(L)$ satisfies the following condition: for each $l \in L$ either $\sigma(l)=l$ or $\sigma^{i}(l) \neq l$ for all $i \geq 1$. Let $\mathcal{R}=L\left(\left(t^{-1} ; \delta\right)\right)$ be the formal pseudo-differential operator ring with $0 \neq \delta \in \operatorname{Der}(L)$. Then the division rings $\mathcal{L}$ and $\mathcal{R}$ satisfy the $c c c$ (Theorem 5.1 and Lemma [7.1). The last fact is due to K. R. Goodearl [14. Many well-known algebras of Gelfand-Kirillov dimension $<3$ are subalgebras of either $\mathcal{L}$ or $\mathcal{R}$, and so satisfy the $c c c$ as well.

Using the result above we prove that the following algebras admit the Dixmier partition and satisfy the commutative centralizer condition and the homogeneous centralizer condition:

1. The first Weyl algebra $A_{1}$. Any noncommutative subalgebra $A$ of the division ring $Q\left(A_{1}\right)$ of the Weyl algebra $A_{1}$ with Gelfand-Kirillov dimension GK $(A)<3$. In particular, the noncommutative deformations of type-A Kleinian singularities are of this type (Corollary 5.3. $)$.

2. The quantum plane $\Lambda=K\langle x, y \mid x y=\lambda y x\rangle, \lambda \in K^{*}$ is not an $i^{\text {th }}$ root of 1 for all $i \geq 1$. Any noncommutative subalgebra $A$ with Gelfand-Kirillov dimension GK $(A)<3$ of the division ring $Q(\Lambda)$ of the quantum plane $\Lambda$ (Corollary 5.4). The fact that $Q(\Lambda)$ satisfies the $c c c$ is due to V. A. Artamonov and P. M. Cohn [2]. The quantum Weyl algebra $A_{1}(\lambda)=K\langle x, y \mid y x-\lambda x y=1\rangle$ is a subalgebra of $Q(\Lambda)$, hence it satisfies the $c c c$, this was proved by V. Mazorchuk [19]. 
3. $(K=\bar{K})$ The ring $\mathcal{D}(X)$ of differential operators on a smooth irreducible algebraic curve $X$. Any noncommutative subalgebra $A$ with Gelfand-Kirillov dimension GK $(A)<3$ of the division ring $Q(\mathcal{D}(X))$ of the algebra $\mathcal{D}(X)$ (Corollary 7.3).

4. The universal enveloping algebra $U s l(2)$ of the Lie algebra $s l(2)$ (note that GK $(U s l(2))=$ $3)$. Any noncommutative subalgebra $A$ of the division ring $Q(U s l(2))$ of the universal enveloping algebra $U \operatorname{sl}(2)$ with Gelfand-Kirillov dimension GK $(A)<4$ and such that $A \cap K(C) \neq K$ where $C$ is the Casimir element of the algebra $U s l(2)$ (Proposition 6.11).

5. The quantum $U_{q} s l(2)$ (note that $\mathrm{GK}\left(U_{q} s l(2)\right)=3$ ). Any noncommutative subalgebra $A$ of the division ring $Q\left(U_{q} s l(2)\right)$ of the algebra $U_{q} s l(2)$ with Gelfand-Kirillov dimension $\operatorname{GK}(A)<4$ and such that $A \cap K(C) \neq K$ where $C \in U_{q} s l(2)$ is the (quantum) Casimir element (Proposition 6.2).

\section{Nonexistence of Nonzero Eigenvalue for Inner Deriva- tions of the Type ad $f(a), f(t) \in K[t], \operatorname{deg} f(t)>1$}

Let $K$ be a field of characteristic zero. In this Section, let $B=\cup_{i \geq 0} B_{i}$ be a filtered $K$-algebra such that the associated graded algebra gr $B=\oplus_{i \geq 0} B_{i} / B_{i-1}$ is a domain, and

$$
\left[B_{i}, B_{j}\right] \subseteq B_{i+j-1} \text { for all } i, j \geq 0, i+j>0 .
$$

In this case, we say that the algebra $B$ has a filtration of nilpotent type, and $\left\{B_{i}\right\}$ is the filtration of nilpotent type. If, in addition, all the elements of the set $B_{0} \backslash Z(B)$ are strongly nilpotent then we say that the algebra $B$ has a filtration of strongly nilpotent type, and $\left\{B_{i}\right\}$ is a filtration of strongly nilpotent type. Note that, in general, the algebra gr $B$ is not necessarily commutative. Although in applications this is usually the case. If $\operatorname{gr} B$ is a commutative domain then the filtration $\left\{B_{i}\right\}$ is of strongly nilpotent type.

For a nonzero element $a \in B$ the unique natural number $n$ such that $a \in B_{n} \backslash B_{n-1}$ is called the degree of the element $a$ (with respect to the filtration) denoted $\operatorname{deg} a(\operatorname{deg} 0:=$ $-\infty)$. The algebra gr $B$ is a domain, thus, for any $a, b \in B$,

$$
\begin{aligned}
\operatorname{deg}(a b) & =\operatorname{deg} a+\operatorname{deg} b, \\
\operatorname{deg}(a+b) & \leq \max \{\operatorname{deg} a, \operatorname{deg} b\}, \\
\operatorname{deg}[a, b] & \leq \operatorname{deg} a+\operatorname{deg} b-1, \quad \text { if } \operatorname{deg} a+\operatorname{deg} b>0 .
\end{aligned}
$$

The first property guarantees that $B$ is a domain since gr $B$ is so.

For a given element $a \in B$, the following three linear maps from $B$ to $B$ commute: the left multiplication $L_{a}$ by the element $a$, the right multiplication $R_{a}$ by the element $a$, and the inner derivation ad $a=L_{a}-R_{a}$ of $B$. So, for each $n \geq 1$,

$$
\left(L_{a}\right)^{n}=\left(\operatorname{ad} a+R_{a}\right)^{n}=\sum_{i=0}^{n}\left(\begin{array}{c}
n \\
i
\end{array}\right)(\operatorname{ad} a)^{i}\left(R_{a}\right)^{n-i} .
$$


Thus, for any $b \in B$,

$$
\begin{aligned}
a^{n} b & =\left(L_{a}\right)^{n} b=\left(\operatorname{ad} a+R_{a}\right)^{n} b=\sum_{i=0}^{n}\left(\begin{array}{c}
n \\
i
\end{array}\right)(\operatorname{ad} a)^{i}(b) a^{n-i} \\
& =\sum_{i=0}^{n} n(n-1) \cdots(n-i+1) \frac{(\operatorname{ad} a)^{i}(b)}{i !} a^{n-i}
\end{aligned}
$$

For a polynomial $f(t) \in K[t]$ we denote by $f^{(i)}(t)$ its $i^{t h}$ derivative $\frac{d^{i} f}{d t^{i}}$ with respect to $t$. If $\operatorname{deg} a>0$ and $\operatorname{deg}_{t}(f)>0$ then it follows from the identity above that

$$
[f(a), b]=\sum_{i \geq 1} \frac{(\operatorname{ad} a)^{i}(b)}{i !} f^{(i)}(a)=(\operatorname{ad} a)(b) f^{\prime}(a)+\cdots
$$

where the dots denote the terms of smaller degree. Moreover,

$$
\operatorname{deg} T_{1}>\operatorname{deg} T_{2}>\cdots, \text { where } T_{i}:=\frac{(\operatorname{ad} a)^{i}(b)}{i !} f^{(i)}(a) .
$$

In fact, if $T_{i} \neq 0$ and $i>1$ then (using $\operatorname{deg} a>0$ )

$$
\begin{aligned}
\operatorname{deg} T_{i} & =\operatorname{deg}(\operatorname{ad} a)^{i}(b)+\operatorname{deg} f^{(i)}(a) \\
& =\operatorname{deg}(\operatorname{ad} a)(\operatorname{ad} a)^{i-1}(b)+\operatorname{deg} f^{(i-1)}(a)-\operatorname{deg} a \\
& \leq \operatorname{deg}(\operatorname{ad} a)^{i-1}(b)+\operatorname{deg}(a)-1+\operatorname{deg} f^{(i-1)}(a)-\operatorname{deg} a \\
& =\operatorname{deg} T_{i-1}-1<\operatorname{deg} T_{i-1} .
\end{aligned}
$$

Theorem 2.1 Let $B=\cup_{i \geq 0} B_{i}$ be a filtered $K$-algebra such that the associated graded algebra gr $B$ is a domain, and $\left[B_{i}, B_{j}\right] \subseteq B_{i+j-1}$ for all $i, j \geq 0, i+j>0$. Let $a$ be an element of the algebra $B$ such that, for some polynomial $f(t) \in K[t]$ of degree $>1$, the inner derivation ad $f(a)$ of the algebra $B$ has a nonzero eigenvalue. Then $a_{0} \in B_{0}$.

Proof. By the assumption, $[f(a), b]=\lambda b$ for some $0 \neq \lambda \in K$ and $0 \neq b \in B$. Without loss of generality we may assume that $\lambda=1$. Suppose that the element $a$ does not belong to $B_{0}$ thus deg $a>0$. We seek a contradiction. By (11),

$$
b=[f(a), b]=\sum_{i \geq 1} \frac{\delta^{i}(b)}{i !} f^{(i)}(a), \text { where } \delta:=\operatorname{ad} a .
$$

Observe that $\delta\left(f^{(i)}(a)\right)=\left[a, f^{(i)}(a)\right]=0$ for all $i$, thus, if we substitute the sum for $b$ above in the right-hand side of the equality above we obtain

$$
b=\sum_{i_{1}, i_{2} \geq 1} \frac{\delta^{i_{1}+i_{2}}(b)}{i_{1} ! i_{2} !} f^{\left(i_{1}\right)}(a) f^{\left(i_{2}\right)}(a) .
$$


Repeating this substitution $s$ times we have

$$
b=\sum_{i_{1}, \ldots, i_{s} \geq 1} \frac{\delta^{i_{1}+\cdots+i_{s}}(b)}{i_{1} ! \cdots i_{s} !} f^{\left(i_{1}\right)}(a) \cdots f^{\left(i_{s}\right)}(a) .
$$

Now, we can find the leading term of the sum above,

$$
b=\delta^{s}(b)\left[f^{\prime}(a)\right]^{s}+\cdots .
$$

The proof of (3) is essentially the same as in the case when $s=1$, and this result follows immediately from the next fact: $\operatorname{let} T_{\left(i_{1}, \ldots, i_{s}\right)}:=\delta^{i_{1}+\cdots+i_{s}}(b) f^{\left(i_{1}\right)}(a) \cdots f^{\left(i_{s}\right)}(a)$; then

$$
\operatorname{deg} T_{\left(i_{1}+1, i_{2}, \ldots, i_{s}\right)}<\operatorname{deg} T_{\left(i_{1}, i_{2}, \ldots, i_{s}\right)}, \text { provided } T_{\left(i_{1}+1, i_{2}, \ldots, i_{s}\right)} \neq 0
$$

since

$$
\begin{aligned}
\operatorname{deg} T_{\left(i_{1}+1, i_{2}, \ldots, i_{s}\right)} & =\operatorname{deg} \delta \delta^{i_{1}+\cdots+i_{s}}(b)+\operatorname{deg} f^{\left(i_{1}+1\right)}(a)+\sum_{\nu \geq 2} \operatorname{deg} f^{\left(i_{\nu}\right)}(a) \\
& \leq \operatorname{deg} \delta^{i_{1}+\cdots+i_{s}}(b)+\operatorname{deg}(a)-1+\operatorname{deg} f^{\left(i_{1}\right)}(a)-\operatorname{deg}(a)+\sum_{\nu \geq 2} \operatorname{deg} f^{\left(i_{\nu}\right)}(a) \\
& =T_{\left(i_{1}, i_{2}, \ldots, i_{s}\right)}-1<\operatorname{deg} T_{\left(i_{1}, i_{2}, \ldots, i_{s}\right)} .
\end{aligned}
$$

Clearly, $\delta^{n}(b) \neq 0$ for all $n \geq 1$ ( since otherwise, by (2), we would have $b=0$, a contradiction). Now, by (3) and since $\operatorname{deg}_{t}\left(f^{\prime}(t)\right) \geq 1, \operatorname{deg}(a) \geq 1$, we obtain

$$
\operatorname{deg} b=\operatorname{deg} \delta^{s}(b)+s \operatorname{deg} f^{\prime}(a) \geq s \operatorname{deg}_{t}\left(f^{\prime}(t)\right) \cdot \operatorname{deg}(a) \geq s, \text { for all } s \geq 1,
$$

which is impossible since $\operatorname{deg} b<\infty$. This contradiction finishes the proof of the theorem.

\section{Proof of Theorem 1.1.}

The associated graded algebra gr $B$ is commutative which implies that the elements $B_{0} \backslash Z(B)$ are strongly nilpotent $\left(\left[B_{i}, B_{j}\right] \subseteq B_{i+j-1}\right.$ for all $i, j \geq 0$, and so $\left.\left(\operatorname{ad} B_{0}\right)^{i+1} B_{i}=0\right)$. Suppose that, for some element $a \in B$ and some polynomial $f(t)$ of degree $>1$, the inner derivation ad $f(a)$ of the algebra $B$ has a nonzero eigenvalue. Then $f(a) \notin Z(B)$ and, by Theorem 2.1 $a \in B_{0}$ thus $f(a) \in B_{0} \backslash Z(B)$ which contradicts to the fact that each element of $B_{0} \backslash Z(B)$ is strongly nilpotent.

Let $C$ be a finitely generated algebra over the field $K$. To each finite set of $K$-algebra generators $x_{1}, \ldots, x_{n}$ one can attach, so-called, the standard filtration of the algebra $C$ (by the total degree of generators):

$$
C_{0}:=K \subseteq C_{1}:=K+\sum_{j=1}^{n} K x_{j} \subseteq \cdots \subseteq C_{i}:=C_{1}^{i} \subseteq \cdots
$$

Definition. A finitely generated algebra $C$ is called an almost commutative algebra if there exists a standard filtration in $C$ such that the associated graded algebra gr $C=$ $\oplus_{i \geq i} C_{i} / C_{i-1}$ is a commutative algebra. 
Each factor algebra of the universal enveloping algebra $U(\mathcal{G})$ of a finite dimensional Lie algebra $\mathcal{G}$ is an almost commutative algebra and vice versa ([21], 8.4.3, or [16]).

Example 1 (Almost Commutative Algebras). Any almost commutative algebra $C$ such that the associated graded algebra gr $C$ is a domain satisfies the conditions of Theorem 1.1 with $C_{0}=K$.

Example 1. $(i)$. The natural filtration of the universal enveloping algebra $U(\mathcal{G})$ is a filtration of strongly nilpotent type since $U(\mathcal{G})_{0}=K$ and the associated graded algebra $\operatorname{gr} U(\mathcal{G})$ is a polynomial algebra in $\operatorname{dim}_{K} \mathcal{G}$ indeterminates.

Example 1. (ii) (the Weyl Algebras). The Weyl algebra $A_{n}$ is an associative algebra generated over the field $K$ by $2 n$ elements $X_{1}, \ldots, X_{n}, \partial_{1}, \ldots, \partial_{n}$ subject to the defining relations:

$$
\begin{gathered}
\partial_{i} X_{i}-X_{i} \partial_{i}=1, \quad i=1, \ldots, n \\
{\left[X_{i}, X_{j}\right]=\left[\partial_{i}, \partial_{j}\right]=\left[\partial_{i}, X_{j}\right]=0, \text { for all } i \neq j .}
\end{gathered}
$$

The Weyl algebra $A_{n}$ is canonically isomorphic to the ring of differential operators

$$
A_{n} \simeq K\left[X_{1}, \ldots, X_{n}, \frac{\partial}{\partial X_{1}}, \ldots, \frac{\partial}{\partial X_{n}}\right], \quad X_{i} \leftrightarrow X_{i}, \quad \partial_{i} \leftrightarrow \frac{\partial}{\partial X_{i}},
$$

with polynomial coefficients $K\left[X_{1}, \ldots, X_{n}\right]$. The field $K$ has characteristic zero, so the centre of the Weyl algebra $A_{n}$ is $K$. The standard filtration of the Weyl algebra $A_{n}$ associated with the canonical generators is called the Bernstein filtration. The associated graded algebra of the Weyl algebra $A_{n}$ with respect to this filtration is a polynomial algebra in $2 n$ indeterminates:

$$
K\left[x_{1}, \ldots, x_{n}, y_{1}, \ldots, y_{n}\right], \quad x_{i}:=X_{i}+K \in A_{n, 1} / A_{n, 0}, \quad y_{i}:=\partial_{i}+K \in A_{n, 1} / A_{n, 0} .
$$

Thus the Bernstein filtration of the Weyl algebra $A_{n}$ is a filtration of strongly nilpotent type.

Corollary 2.2 Let a be a non-scalar element of the Weyl algebra $A_{n}$ over a field $K$ of characteristic zero. Then, for an arbitrary polynomial $f(t) \in K[t]$ of degree $>1,0$ is the only eigenvalue of the inner derivation ad $f(a)$ of $A_{n}$.

An arbitrary $K$-derivation of the Weyl algebra $A_{n}$ over a field of characteristic zero is an inner derivation [10, so Corollary 2.2] is a statement about derivations of the Weyl algebra. In particular, if an (inner) derivation $\delta=\operatorname{ad} b$ of the Weyl algebra $A_{n}$ has a nonzero eigenvalue then the element $b \in A_{n}$ is not a polynomial $f(a)$ of any element $a \in A_{n}$.

Example 2 (Rings of Differential Operators on Smooth Algebraic Varieties). Let $R$ be a commutative algebra over a field $K$ of characteristic zero. The ring of differential operators $\mathcal{D}(R)=\cup_{i \geq 0} \mathcal{D}(R)_{i}$ with coefficients from the algebra $R$ is a positively filtered subalgebra of the algebra $\operatorname{End}_{K}(R)$ of $K$-linear maps in $R$ defined by the rule

$$
\mathcal{D}(R)_{0}:=\left\{f \in \operatorname{End}_{K}(R) \mid f r-r f=0 \text { for all } r \in R\right\}=\operatorname{End}_{R}(R)=R
$$


and

$$
\mathcal{D}(R)_{i}:=\left\{f \in \operatorname{End}_{K}(R) \mid f r-r f \in \mathcal{D}(R)_{i-1} \text { for all } r \in R\right\} .
$$

For a smooth irreducible affine variety $X$ over the field $K$ its coordinate algebra $R=\mathcal{O}(X)$ is a commutative affine regular domain. In this case, the ring of differential operators $\mathcal{D}(X):=\mathcal{D}(\mathcal{O}(X))$ on $X$ is generated, as a $K$-subalgebra of $\operatorname{End}_{K}(\mathcal{O}(X))$, by the coordinate algebra $\mathcal{O}(X)$ and the $\mathcal{O}(X)$-module $\operatorname{Der}_{K}(\mathcal{O}(X))$ of $K$-derivations of the algebra $\mathcal{O}(X)(21], 15.5 .6)$. The associated graded algebra gr $\mathcal{D}(X)=\oplus_{i \geq 0} \mathcal{D}(X)_{i} / \mathcal{D}(X)_{i-1}$ is a commutative (affine) domain, 21], 15.4.7.

Corollary 2.3 Let $\mathcal{G}$ be a nilpotent finite dimensional Lie algebra over a field $K$ of characteristic zero, $A$ be a noncommutative prime factor ring of its universal enveloping algebra $U(\mathcal{G})$, and $a \in A$ be an arbitrary element. Then, for an arbitrary polynomial $f(t) \in K[t]$ of degree $>1,0$ is the only eigenvalue of the inner derivation ad $f(a)$ of $A$.

Proof. The algebra $A$ is a domain such that the localization $S^{-1} A$ of the algebra $A$ at $S:=Z(A) \backslash\{0\}$ is the Weyl algebra $A_{n}(Q)$ with coefficients from the field $Q:=S^{-1} Z(A)$ of quotients of the centre $Z(A)$ of $A$ ([21, 14.6.9.(i)). Now, the result follows from Corollary 2.2 .

We denote by $A_{1}^{\prime}$ the localization of the Weyl algebra $A_{1}$ at the powers of the element $x$. Since $A_{1}=\mathcal{D}(K[x])$, we have $A_{1}^{\prime}=\mathcal{D}\left(K\left[x, x^{-1}\right]\right)$. For a natural number $m$, let $A_{m}^{\prime}:=A_{1}^{\prime} \otimes \cdots \otimes A_{1}^{\prime}, m$ times.

Corollary 2.4 Let $A=C \otimes A_{n} \otimes A_{m}^{\prime}$ where $C$ is a commutative algebra and $n, m \geq 1$, and let $a \in A$. Then, for an arbitrary polynomial $f(t) \in K[t]$ of degree $>1,0$ is the only eigenvalue of the inner derivation ad $f(a)$ of $A$.

Proof. Note that the algebra $A$ is a domain. On the one hand, the algebra $A$ is the Weyl algebra $A_{n}(\Lambda)$ with coefficients from the algebra $\Lambda:=C \otimes A_{m}^{\prime}$. The Bernstein filtration $A_{n}(\Lambda)=\cup_{i \geq 0} A_{n}(\Lambda)_{i}$ for the Weyl algebra $A_{n}(\Lambda)$ is a filtration of nilpotent type since $\operatorname{gr} A_{n}(\Lambda)$ is isomorphic to the polynomial algebra $\Lambda\left[x_{1}, \ldots, x_{n}, y_{1}, \ldots, y_{n}\right]$ in $2 n$ indeterminates with coefficients from the algebra $\Lambda$.

On the other hand, the algebra $A=\Lambda^{\prime} \otimes A_{m}^{\prime}=\Lambda^{\prime} \otimes \mathcal{D}(Q)$ where $\Lambda^{\prime}:=C \otimes A_{n}$ and $Q:=K\left[z_{1}, z_{1}^{-1}, \ldots, z_{m}, z_{m}^{-1}\right]$ is the Laurent polynomial algebra. The order filtration $A_{m}^{\prime}=\mathcal{D}(Q)=\cup_{i \geq 0} \mathcal{D}(Q)_{i}$ of the algebra $A_{m}^{\prime}$ can be extended to the filtration $A=\cup_{i \geq 0} B_{i}$, $B_{i}:=\Lambda^{\prime} \otimes \mathcal{D}(Q)_{i}$, for the algebra $A$. The filtration $\left\{B_{i}\right\}$ is of nilpotent type since

$$
\operatorname{gr} A \simeq \Lambda^{\prime} \otimes \operatorname{gr} \mathcal{D}(Q) \simeq \Lambda^{\prime} \otimes K\left[z_{1}, z_{1}^{-1}, \ldots, z_{m}, z_{m}^{-1}, y_{1}, \ldots, y_{m}\right]
$$

Note that $A_{n}(\Lambda)_{0}=\Lambda=C \otimes A_{m}^{\prime}, B_{0}=\Lambda^{\prime} \otimes \mathcal{D}(Q)_{0}=C \otimes A_{n} \otimes Q$, and $I:=A_{n}(\Lambda)_{0} \cap B_{0}=$ $C \otimes Q$. Each element of the set $I \backslash Z(A)$ is a strongly nilpotent element of the algebra $A$.

Suppose that, for some element $a \in A$ and some polynomial $f(t)$ of degree $>1$, the inner derivation ad $f(a)$ of the algebra $A$ has a nonzero eigenvalue. Then $f(a) \notin Z(A)$ and, by Theorem 2.1], $a \in I$, a contradiction.

Recall that an algebraic Lie algebra $\mathcal{G}$ is the Lie algebra of a (linear) affine algebraic group. The reader is referred to the books [8] and [21] for detail. 
Corollary 2.5 Let $\mathcal{G}$ be a completely solvable, algebraic Lie algebra over a field $K$ of characteristic zero, $A$ be a prime factor algebra of the universal enveloping algebra $U(\mathcal{G})$ and $a$ be an arbitrary element of the algebra $A$. Then, for an arbitrary polynomial $f(t) \in K[t]$ of degree $>1,0$ is the only eigenvalue of the inner derivation ad $f(a)$ of $A$.

Proof. By [21], 14.2.11, $A$ is a domain. By 21], 14.7.6, the localization $A_{e}$ of the algebra $A$ at the powers of the particularly chosen element $e \in A$ is the algebra from Corollary 2.4. $A_{e} \simeq Z\left(A_{e}\right) \otimes A_{n} \otimes A_{m}^{\prime}$. So, the result follows from Corollary 2.4.

Let $A$ be an algebra. A left $A$-module $M$ is called faithful if $a M=0$ implies $a=0$ for any $a \in A$. If an algebra $A$ has a left faithful simple module then $A$ is called (left) primitive.

Corollary 2.6 Let $\mathcal{G}$ be a completely solvable Lie algebra over a field $K$ of characteristic zero, $A$ be a prime factor algebra of the universal enveloping algebra $U(\mathcal{G})$, and $a \in A$. Then, for an arbitrary polynomial $f(t) \in K[t]$ of degree $>1,0$ is the only eigenvalue of the inner derivation ad $f(a)$ of $A$.

Proof. By [21], 14.2.11, $A$ is a domain. Combining [21, 14.9.16 and 14.8.11, $A$ is a subalgebra of the algebra from Corollary 2.4 with $C$ a polynomial algebra. Now, the result follows from Corollary 2.4

Remark. The same result holds for the algebra $\mathcal{A}(V, \delta, \Gamma)$ from [21], Ch. 14, Section 8 (since, by [21], 14.8.11, $\mathcal{A}(V, \delta, \Gamma)$ is a subalgebra of the algebra from Corollary 2.4 with $C$ is a polynomial algebra).

Corollary 2.7 Let the algebra $B$ be as in Theorem 1.1, and let $a \in B \backslash Z(B)$. If $f(a) \in$ $Z(B)$ for some polynomial $f(t) \in K[t]$ of degree $>1$ then 0 is the only eigenvalue for the inner derivation $\operatorname{ad}(a)$, and $a \in B_{0}$. If, in addition, $B_{0} \subseteq Z(B)$ then $f(x) \in Z(B), x \in B$, imply $x \in Z(B)$.

Proof. $\lambda b=[a, b]=[f(a)+a, b], 0 \neq b \in B, \lambda \in K$, imply $\lambda=0$, by Theorem 1.1

Suppose that $a \notin B_{0}$. Then, by (II), $0=[f(a), b]=[a, b] f^{\prime}(a)+\cdots$ for all $b \in B$, hence $[a, b]=0$ for all $b \in B$, that is $a \in Z(B)$, a contradiction. Therefore, $a \in B_{0}$. Then the last statement is obvious.

\section{Noncommutative Domains of Gelfand-Kirillov $<3$ Admit the Dixmier Partition}

In this section, if it is not stated otherwise, $K$ is an algebraically closed field of characteristic zero, and a $K$-algebra $A$ is a noncommutative domain.

The aim of this section is to prove that the algebra $A$ admits the Dixmier partition provided that its Gelfand-Kirillov dimension is less than 3 (Corollary 3.2). 
For each non-central element $a \in A \backslash Z(A)$, we have the corresponding nonzero inner derivation ad $a$ of the algebra $A$. Its torsion algebra

$$
F(a)=\bigoplus_{\lambda \in K} F(a, \lambda), \quad F(a, \lambda)=\cup_{n \geq 0} F(a, \lambda, n),
$$

where $F(a, \lambda, n):=\operatorname{ker}(\operatorname{ad} a-\lambda)^{n+1}=\left\{x \in A \mid(\operatorname{ad} a-\lambda)^{n+1} x=0\right\}$. For any $x, y \in A$, $\lambda, \mu \in K$, and $n \geq 1$, the identity

$$
(\operatorname{ad} a-(\lambda+\mu))^{n}(x y)=\sum_{i=0}^{n}\left(\begin{array}{c}
n \\
i
\end{array}\right)(\operatorname{ad} a-\lambda)^{i}(x) \cdot(\operatorname{ad} a-\mu)^{n-i}(y)
$$

implies that

$$
F(a, \lambda, n) \cdot F(a, \mu, m) \subseteq F(a, \lambda+\mu, n+m) \text { for all } \lambda, \mu \in K, n, m \geq 0 .
$$

It follows that the nil-algebra

$$
N(a)=F(a, 0)=\cup_{n \geq 0} N(a, n), \quad N(a, n)=F(a, 0, n),
$$

is a positively filtered algebra, and that the eigenvalue algebra

$$
D(a)=\bigoplus_{\lambda \in \operatorname{Ev}(\operatorname{ad} a)} D(a, \lambda), \quad D(a, \lambda)=F(a, \lambda, 0),
$$

is an $\operatorname{Ev}(\operatorname{ad} a)$-graded algebra $(D(a, \lambda) D(a, \mu) \subseteq D(a, \lambda+\mu)$ for all $\lambda, \mu \in \operatorname{Ev}(\operatorname{ad} a))$ and the set $\operatorname{Ev}(\operatorname{ad} a)$ is an additive submonoid of the field $K$ since the algebra $A$ is a domain.

For an element $0 \neq x \in F(a, \lambda)$, let $\pi(x)$ be the unique natural number $n$ such that $x \in F(a, \lambda, n) \backslash F(a, \lambda, n-1)$. If $0 \neq y \in F(a, \mu)$ then

$$
\pi(x y)=\pi(x)+\pi(y)
$$

since by (6),$\pi(x y) \leq \pi(x)+\pi(y)$ and, by (5),

$$
(\operatorname{ad} a-(\lambda+\mu))^{\pi(x)+\pi(y)}(x y)=\left(\begin{array}{c}
\pi(x)+\pi(y) \\
\pi(x)
\end{array}\right)(\operatorname{ad} a-\lambda)^{\pi(x)}(x) \cdot(\operatorname{ad} a-\mu)^{\pi(y)}(y) \neq 0,
$$

since $A$ is a domain and char $K=0$.

Lemma 3.1 Let $A$ be a noncommutative domain, and let $a \in A \backslash Z(A)$.

1. $K[a] \subseteq C(a)$, and so $\mathrm{GK}(C(a)) \geq 1$.

2. Suppose that $F(a, \lambda) \neq D(a, \lambda)$ for some $0 \neq \lambda \in K$. Then the Gelfand-Kirillov dimension of the $K$-algebra $B:=\oplus_{n \geq 0} F(a, n \lambda)$ is not less than 3 .

3. If $F(a) \neq C(a)$ then $\operatorname{GK}(F(a)) \geq 2$. 
Proof. The first statement is evident since the algebra $A$ is a domain and $K=\bar{K}$.

2. Choose an element $x \in F(a, \lambda, 1) \backslash D(a, \lambda)$ then $0 \neq y:=(\operatorname{ad} a-\lambda) x \in D(a, \lambda)$. Note that $a^{i} y^{j} x^{k} \in F(a,(j+k) \lambda, k) \backslash F(a,(j+k) \lambda, k-1)$ for all $i, j, k \geq 0$ with $j+k>0$. This implies that

$$
B \supseteq \sum_{i, j, k \geq 0} K a^{i} y^{j} x^{k}=\bigoplus_{i, j, k \geq 0} K a^{i} y^{j} x^{k}
$$

hence $\operatorname{GK}(B) \geq 3$.

3. If $F(a) \neq C(a)$ then either $N(a) \neq C(a)$ or $D(a) \neq C(a)$ (or both). In the first (resp. second) case, we can choose an element $x \in N(a, 1) \backslash C(a)$ (resp. $0 \neq y \in D(a, \lambda)$ for some $0 \neq \lambda \in \operatorname{Ev}(\operatorname{ad} a))$. Then

$$
F(a) \supseteq \sum_{i, j \geq 0} K a^{i} x^{j}=\bigoplus_{i, j \geq 0} K a^{i} x^{j} \quad\left(\text { resp. } \quad F(a) \supseteq \sum_{i, j \geq 0} K a^{i} y^{j}=\bigoplus_{i, j \geq 0} K a^{i} y^{j}\right) .
$$

In both cases, this implies that $\mathrm{GK}(F(a)) \geq 2$.

Corollary 3.2 Let a $K$-algebra $A$ be a noncommutative domain of Gelfand-Kirillov dimension $<3$, and let $a \in A \backslash Z(A)$.

1. $F(a, \lambda)=D(a, \lambda)$ for each $\lambda \in K$.

2. Either $F(a)=N(a)$ or $F(a)=D(a)$.

3. The algebra $A$ admits the Dixmier partition.

Proof. 1. This follows from Lemma 3.1 and the fact that $\mathrm{GK}(A)<3$.

2. By statement 1 ,

$$
F(a)=N(a) \bigoplus\left(\bigoplus_{0 \neq \lambda \in \operatorname{Ev}(\operatorname{ad} a)} D(a, \lambda)\right)=N(a)+D(a) .
$$

If $F(a) \neq N(a)$ and $F(a) \neq D(a)$ then $N(a, 1) \neq N(a, 0)$ and $D(a, \lambda) \neq 0$ for some $0 \neq \lambda \in$ $\operatorname{Ev}(\operatorname{ad} a)$. The algebra $A$ is a domain, so $0 \neq N(a, 1) D(a, \lambda)$, and so $F(a, \lambda, 1) \neq F(a, \lambda, 0)$ by (17) which contradicts to the first statement.

3. The statements 2 and 3 are equivalent.

Suppose that the field $K$ is not necessarily algebraically closed but still has characteristic zero. Let a $K$-algebra $A$ be a noncommutative domain. Via the $K$-algebra monomorphism $A \rightarrow \bar{A}:=\bar{K} \otimes A, a \rightarrow 1 \otimes a$, we can identify the algebra $A$ with its image in $\bar{A}$. The next result follows from Corollary 3.2 (since the type of elements remain the same under field extension).

Corollary 3.3 Let $K$ be a field of characteristic zero (not necessarily algebraically closed), and let a $K$-algebra $A$ be a noncommutative domain of Gelfand-Kirillov dimension $<3$ such that the algebra $\bar{A}=\bar{K} \otimes A$ is a domain. Then the algebra $A$ admits the Dixmier partition. 


\section{Algebras that Satisfy the Commutative Centralizer Condition, and Dixmier's Problem 6}

Definition. A noncommutative algebra $A$ satisfies the commutative centralizer condition (the $c c c$, for short) if the centralizer of each element of the set $A \backslash Z(A)$ is a commutative algebra.

Clearly, every noncommutative subalgebra of an algebra that satisfies the commutative centralizer condition has this property as well.

Example 1. The Weyl algebra $A_{1}$ satisfies the commutative centralizer condition [1].

Example 2. The formal pseudo-differential operator ring $L\left(\left(t^{-1} ; \delta\right)\right)$ with coefficients from a field extension $L / K$ satisfies the commutative centralizer condition where a derivation $\delta$ of $L$ has $\operatorname{ker} \delta=K$ (Lemma 7.1).

Example 3. The skew Laurent series algebra $L=K(H)\left[\left[X, X^{-1} ; \sigma\right]\right], \sigma(H)=H-1$, satisfies the isotropic centralizer condition. The Weyl algebra $A_{1}$, its ring of quotients (so-called, the first Weyl skew field), and the noncommutative deformations of type-A Kleinian singularity are subalgebras of $L$, and so they satisfy the isotropic centralizer condition (Section 5).

Example 4. The skew Laurent series algebra $R=K(H)\left[\left[X, X^{-1} ; \tau\right]\right], \tau(H)=\lambda H$, $\lambda \in K^{*}$ is not a root of 1 , satisfies the commutative centralizer condition. The quantum plane $\Lambda$ and the quantum Weyl algebra $A_{1}(\lambda)$, and their rings of quotients are subalgebras of $R$, and so they satisfy the commutative centralizer condition (Section 5 ).

Lemma 4.1 Let $C$ be a commutative subalgebra of an algebra $A$ that satisfies the commutative centralizer condition. The following statements are equivalent.

1. $C$ is a maximal commutative subalgebra of $A$.

2. There exists an element $a \in A \backslash Z(A)$ such that $C=C(a)$.

3. $C \neq Z(A)$ and $C=C(b)$, for all elements $b \in C \backslash Z(A)$.

So, for each element $a \in A \backslash Z(A)$, its centralizer $C(a)$ is the unique maximal commutative subalgebra that contains the element $a$.

Proof. $(1 \Rightarrow 3)$ Let $C$ be a maximal commutative subalgebra of $A$. Then $Z(A) \subseteq C$, but $Z(A) \neq C$ since $A$ is a noncommutative algebra. The centralizer $C(b)$ of each element $b \in C \backslash Z(A)$ contains $C$. Then $C=C(b)$ since $C(b)$ is an commutative algebra and $C$ is a maximal commutative subalgebra of $A$.

$(3 \Rightarrow 2)$ Evident.

$(2 \Rightarrow 1)$ Let $C=C(a)$, for some $a \in A \backslash Z(A)$, and let $C^{\prime}$ be a commutative subalgebra of $A$ containing $C$. Then $C^{\prime} \subseteq C$ since $a \in C^{\prime}$ and $\left[a, C^{\prime}\right]=0$. The algebra $A$ satisfies the ccc, hence $C$ is the maximal commutative subalgebra of $A$.

So, Lemma 4.1 states that for an algebra that satisfies the $c c c$ the concepts of centralizer and maximal commutative subalgebra coincide. 
Corollary 4.2 Let an algebra A satisfy the commutative centralizer condition.

1. Let $a, b \in A \backslash Z(A)$. Then $a b=b a$ iff $C(a)=C(b)$. $a b \neq b a$ iff $C(a) \cap C(b)=Z(A)$.

2. Let $C$ be a maximal commutative subalgebra of $A, a \in A \backslash Z(A)$, and $p(t) \in Z(A)[t]$ be a polynomial of positive degree with coefficients from $Z(A)$. If $p(a) \in C \backslash Z(A)$ then $a \in C$.

Proof. 1. If $C(a)=C(b)$ then $[a, b]=0$ since $A$ satisfies the $c c c$. Suppose that the elements $a$ and $b$ commute. By Lemma 4.1.(2), $C(a)$ is a maximal commutative subalgebra of $A$. Since $b \in C(a) \backslash Z(A)$, by Lemma 4.1.(3), $C(a)=C(b)$. This proves the first part of the first statement.

If $a b \neq b a$ then $C(a) \cap C(b)=Z(A)$ since otherwise we can choose an element, say $c$, from the set $(C(a) \cap C(b)) \backslash Z(A)$. By Lemma 4.1.(3), $C(a)=C(b)=C(c)$, a contradiction.

If $C(a) \cap C(b)=Z(A)$ then $a b \neq b a$ since otherwise by the first statement $C(a)=C(b)$, hence $C(a)=C(a) \cap C(b)=Z(A)$ which contradicts to the fact that $a \notin Z(A)(a \in C(a))$.

2. By Lemma 4.1.(3), $C=C(b)$ where $b=p(a)$. The elements $a$ and $b$ satisfy the condition of statement 1 , so $C(a)=C(b)$, hence $a \in C$.

Lemma 4.3 Let an algebra A satisfy the commutative centralizer condition, and let $a, b$, and $p$ be nonzero elements of $A$ such that $a b=b a,[a, p]=\lambda p$, and $[b, p]=\mu p$ for some $\lambda, \mu \in K^{*}$. Then $b \in K^{*} a+Z(A)$.

Proof. The assumptions $[a, p]=\lambda p \neq 0$ and $[b, p]=\mu p \neq 0$ imply that $a, b, p \notin Z(A)$. Since the elements $a$ and $b$ commute and do not belong to the centre of the algebra $A$, we have $C:=C(a)=C(b)$ and $C \cap C(p)=Z(A)$ (Corollary 4.2). It follows from $[\mu a-\lambda b, p]=0$ that $\mu a-\lambda b \in C \cap C(p)=Z(A)$, hence $b \in K^{*} a+Z(A)$.

Let $A$ be a noncommutative algebra. Suppose that the centralizer $C(a)$ of an element $a \in A$ is a commutative algebra. Then $a \notin Z(A)$ since $A$ is a noncommutative algebra. Suppose that the set $D(a, \lambda, A) \neq 0$, for some $0 \neq \lambda \in K$, contains an invertible element, say $p$, then $[a, p]=\lambda p$. This equality is equivalent to the equality $p a=(a-\lambda) p$. Then $\left[a, p^{-1}\right]=-\lambda p^{-1}$, and so $D(a,-\lambda, A) \neq 0$. The inner automorphism $\omega_{p}: u \rightarrow p u p^{-1}$ of the algebra $A$ preserves the centralizer $C(a)$, that is $\omega_{p}(C(a)) \subseteq C(a)$, since, for all $c \in C(a)$,

$$
p c p^{-1} a=p c(a+\lambda) p^{-1}=p(a+\lambda) c p^{-1}=(a-\lambda+\lambda) p c p^{-1}=a p c p^{-1} .
$$

In the argument above we use the commutativity of the centralizer $C(a)$. The inner automorphism $\omega_{p^{-1}}$ also preserves the algebra $C(a)$, thus

$$
\omega_{p}(C(a))=C(a)
$$

If $q$ is another nonzero element of $D(a, \lambda, A)$ then $q p^{-1} \in C(a)$ since

$$
q p^{-1} a=q(a+\lambda) p^{-1}=(a-\lambda+\lambda) q p^{-1}=a q p^{-1} .
$$


This proves that

$$
D(a, \lambda, A)=C(a) p=p C(a) .
$$

We denote by $\tau_{\lambda}$ the restriction of the inner automorphism $\omega_{p}$ of the algebra $A$ to the subalgebra $C(a)$. Since the algebra $C(a)$ is commutative,

$$
q c=\tau_{\lambda}(c) q, \text { for all } 0 \neq q \in D(a, \lambda, A), c \in C(a) .
$$

Proposition 4.4 Suppose that an algebra A satisfies the commutative centralizer condition. Let $a, b, p$, and $q$ be nonzero elements of the algebra $A$ such that $a b=b a,[a, p]=\lambda p$, and $[b, q]=\mu q$ for some $\lambda, \mu \in K^{*}$. If the elements $p$ and $q$ are invertible in $A$, the centralizer $C(a)$ of the element $a$ is a domain, and none of the elements $p^{i}, i \geq 1$, is an eigenvector for $\operatorname{ad} b$ then the sum $M:=\sum_{i, j \geq 0} C(a) q^{i} p^{j}$ is a free left $C(a)$-submodule of $A$ with the free basis $\left\{q^{i} p^{j} \mid i, j \geq 0\right\}$.

Proof. The elements $a$ and $b$ commute and do not belong to the centre of the algebra $A$ since $[a, p]=\lambda p \neq 0$ and $[b, q]=\mu q \neq 0$. The algebra $A$ satisfies the $c c c$, so $C:=$ $C(a)=C(b)$ is a commutative subalgebra of $A$ which is a domain (by the assumption). The elements $p$ and $q$ are units in $A$, so, by (8),$D(a, \lambda, A)=C p=p C$ and $D(b, \mu, A)=$ $C q=q C$. We denote by $\tau_{\lambda}$ and $\tau_{\mu}$ the restrictions of the inner automorphisms $\omega_{p}$ and $\omega_{q}$ of the algebra $A$ to the subalgebra $C$ as defined in (9). The element $q$ is a unit in $A$, so $0 \neq q^{i} \in D(b, i \mu, A)$, for all $i \geq 0$, and $\{i \mu\}$ are distinct eigenvalues of the inner derivation ad $b$ of the algebra $A$ since the field $K$ has characteristic zero. Thus

$$
N:=\sum_{i \geq 0} C q^{i}=\bigoplus_{i \geq 0} C q^{i}=\bigoplus_{i \geq 0} q^{i} C
$$

is the free left and right $C$-module since $q^{i} C=C q^{i}$. Moreover, $q^{i} c=\tau_{\mu}^{i}(c) q^{i}$, for all $c \in C$.

For an element $u=\sum c_{i} q^{i}$ of $N$ with $c_{i} \in C$, the set $\operatorname{supp} u:=\left\{i \mid c_{i} \neq 0\right\}$ is called the support of $u$. If $i \in \operatorname{supp} u$ then

$$
|\operatorname{supp}(\operatorname{ad} b-i \mu)(u)|=|\operatorname{supp} u|-1 .
$$

Suppose that the set $\left\{q^{i} p^{j} \mid i, j \geq 0\right\}$ is not a free basis for the left $C$-module $N$. Then there exists a nontrivial relation,

$$
r:=u_{d} p^{d}+\cdots+u_{1} p+u_{0}=0 \text { with } u_{i} \in N,
$$

where $d \geq 1$ and $u_{d} \neq 0$ (see the definition of the $C$-module $N$ ). The number $d$ is called the degree of the relation $r$. We may assume that $d \geq 1$ is the least possible degree for all nontrivial relations, then $u_{0} \neq 0$, since otherwise we can divide the relation $r$ by $p$ on the right and obtain a nontrivial relation of degree $d-1$, a contradiction. We may also assume that the number $s=\left|\operatorname{supp} u_{0}\right|$ is the least possible for all nontrivial relations of degree $d$. The element $b$ belongs to the algebra $C$, so, for each $j \geq 1$,

$$
(\operatorname{ad} b)\left(p^{j}\right)=b p^{j}-p^{j} b=\left(b-\tau_{\lambda}^{j}(b)\right) p^{j}=b_{j} p^{j},
$$


where $b_{j}:=b-\tau_{\lambda}^{j}(b) \in C$. Since $(\operatorname{ad} a)\left(p^{j}\right)=j \lambda p^{j}$, and, by the assumption, for each $j \geq 1$, the element $p^{j}$ is not an eigenvector for ad $b$, we conclude that each element $b_{j}$ does not belong to the field $K$. Let us fix $k \in \operatorname{supp} u_{0}$, then

$$
\left|\operatorname{supp}(\operatorname{ad} b-k \mu)\left(u_{0}\right)\right|<\left|\operatorname{supp} u_{0}\right|
$$

and so the relation

$$
(\operatorname{ad} b-k \mu)(r)=\sum_{j=1}^{d}\left((\operatorname{ad} b-k \mu)\left(u_{j}\right)+u_{j} b_{j}\right) p^{j}+(\operatorname{ad} b-k \mu)\left(u_{0}\right)=0
$$

must be trivial by the minimality of $d$ and $s$. This means that for each $j$ the coefficient of $p^{j}$ must be zero. In particular, for $j=d$, we have

$$
(\operatorname{ad} b-k \mu)\left(u_{d}\right)+u_{d} b_{d}=0 .
$$

The element $u_{d}$ has the form $\sum c_{i} q^{i}$ where not all $c_{i} \in C$ are equal to zero. Substituting the sum $u_{d}=\sum c_{i} q^{i}$ in the equality above and using $(\operatorname{ad} b)\left(q^{i}\right)=i \mu q^{i}$, we obtain the equality

$$
0=\sum\left((i-k) \mu c_{i} q^{i}+c_{i} q^{i} b_{d}\right)=\sum c_{i}\left((i-k) \mu+\tau_{\mu}^{i}\left(b_{d}\right)\right) q^{i}
$$

By (10), for each $i$, the coefficient of $q^{i}$ must be 0 . Since not all elements $c_{i}$ are zero and $C$ is a domain (by the assumption), we must have, for some $i$ ( $\operatorname{cuch}$ that $c_{i} \neq 0$ ),

$$
(i-k) \mu+\tau_{\mu}^{i}\left(b_{d}\right)=0
$$

hence $b_{d} \in K$, a contradiction. Thus $M$ is a free left $C$-module with the basis $\left\{q^{i} p^{j}\right\}$.

Recall that commuting non-central elements of an algebra that satisfies the $c c c$ have common centralizer (Corollary 4.2.(1)). The next result shows that they also have common nil-algebra.

Proposition 4.5 Suppose that an algebra A satisfies the commutative centralizer condition, and that elements $a, b \in A \backslash Z(A)$ commute. Then $N(a, A)=N(b, A)$, and $N(a, n, A)=$ $N(b, n, A)$ for all $n \geq 0$.

Proof. It suffices to prove that $N(a, n, A)=N(b, n, A)$ for all $n \geq 0$. We use induction on $n$. The algebra $A$ satisfies the commutative centralizer condition, and the non-central elements $a$ and $b$ commute, hence $C(a)=C(b)$ (Corollary 4.2), and the case $n=0$ holds. The derivations $\partial:=\operatorname{ad} a$ and $\delta:=\operatorname{ad} b$ commute $(\partial \delta-\delta \partial=\operatorname{ad}[a, b]=\operatorname{ad} 0=0)$. Suppose that $N(a, i, A)=N(b, i, A)$ for all $i \leq n$. Then

$$
\begin{aligned}
f \in N(a, n+1, A) & \Leftrightarrow \quad 0=\partial^{n+2} f=\partial^{n+1} \partial f \Leftrightarrow \partial f \in N(a, n, A)=N(b, n, A) \Leftrightarrow \\
0=\delta^{n+1} \partial f=\partial \delta^{n+1} f & \Leftrightarrow \quad \delta^{n+1} f \in C(a, A)=C(b, A) \Leftrightarrow 0=\delta \delta^{n+1} f=\delta^{n+2} f,
\end{aligned}
$$


thus $N(a, n+1, A)=N(b, n+1, A)$. By induction, $N(a, n, A)=N(b, n, A)$ for all $n \geq 0$.

\section{Proof of Theorem 1.3 .}

The $K$-algebra $A$ has Gelfand-Kirillov dimension $<3$, and $\bar{A}$ is a domain, so $A$ admits the Dixmier partition (Corollary 3.3). The algebra $A$ satisfies the $c c c$ as a noncommutative subalgebra of the algebra $\Gamma$ that satisfies the $c c c$. So, it remains to show that the algebra $A$ satisfies the $h c c$. Let $a \in A \backslash Z(A)$, and let $C=C(a)$.

Suppose that the algebra $C$ contains a strongly nilpotent element (resp. a weakly nilpotent element). Then, by Proposition 4.5, all the elements of $C \backslash Z(A)$ are strongly nilpotent (resp. weakly nilpotent).

Suppose that $C$ doest not contain a nilpotent element. Then either all the elements of $C \backslash Z(A)$ are generic or, otherwise, there exists a semi-simple element, say $a \in C \backslash Z(A)$. Note that the field extension does not change the type of elements, $\operatorname{GK}_{\bar{K}}(\bar{A})=\mathrm{GK}(A)<3$, $Z(\bar{A})=\bar{K} \otimes Z(A)$, and $\bar{A}$ is a domain. So, first let us assume that $K=\bar{K}$. Suppose that $b$ is another semi-simple element of $C$. Then $a b=b a(C$ is a commutative algebra since the algebra $A$ satisfies the $c c c),[a, p]=\lambda p$, and $[b, q]=\mu q$ for some non-zero elements $p, q \in A$ and some nonzero scalars $\lambda, \mu \in K^{*}$. We claim that, for some $i \geq 1$, the element $p^{i}$ is an eigenvector for the inner derivation ad $b$ of the algebra $A$. Suppose that this is not true, then, by Proposition 4.4 the sum $M=\sum_{i, j \geq 0} C q^{i} p^{j}$ is a free left $C$-submodule of $A$ with the free basis $\left\{q^{i} p^{j} \mid i, j \geq 0\right\}$. The element $a$ is not an algebraic element (since $a \notin K$ and $K=\bar{K}$ ), so the subalgebra $K[a]$ of $C$ generated by the element $a$ is isomorphic to a polynomial algebra in one indeterminate. Let $\Lambda$ be the $K$-subalgebra of $A$ generated by the elements $a, p$, and $q$, and let $\left\{\Lambda_{n}\right\}_{n \geq 0}$ be the standard filtration of the algebra $\Lambda$ determined by the total degree of the (non-commutative) generators. For each $n \geq 0$,

$$
\Lambda_{n} \supseteq \bigoplus_{i, j, k \geq 0}\left\{K a^{i} q^{j} p^{k} \mid i+j+k \leq n\right\},
$$

and so

$$
\operatorname{dim}_{K} \Lambda_{n} \geq\left(\begin{array}{c}
n+3 \\
3
\end{array}\right)=\frac{(n+1)(n+2)(n+3)}{3 !},
$$

hence the Gelfand-Kirillov dimension $\operatorname{GK}(\Lambda) \geq 3$. On the other hand, $\Lambda$ is a subalgebra of the algebra $A$, and so $\operatorname{GK}(\Lambda) \leq \mathrm{GK}(A)<3$, a contradiction. We have proved that, for some $i \geq 1$, the element $p^{i}$ is a common eigenvector for the inner derivations ad $a$ and ad $b:\left[a, p^{i}\right]=i \lambda p^{i} \neq 0$ and $\left[b, p^{i}\right]=\gamma p^{i}$ for some $\gamma \in K$. We must have $\gamma \neq 0$ since otherwise the non-central elements $b$ and $p^{i}$ commute, and so they have the common centralizer $C(b)=C\left(p^{i}\right)$. By the assumption, the non-central elements $a$ and $b$ commute, so $C(a)=C(b)$ which implies that the elements $a$ and $p^{i}$ commute which is impossible. Now, by Lemma 4.3, $b \in K^{*} a+Z(A)$, hence $K^{*} a+Z(A)$ is the set of all semi-simple elements of $C$ (where $K=\bar{K}$ ).

Suppose that $K \neq \bar{K}$. Since $a \in A, C(a, \bar{A})=\bar{K} \otimes C(a, A), Z(\bar{A})=\bar{K} \otimes Z(A)$, and $\bar{K}^{*} a+Z(\bar{A})$ is the set of all the semi-simple elements in $C(a, \bar{A})$, we see that $K^{*} a+Z(A)$ is the set of all the semi-simple elements in $C(a, A)$. So, the algebra $A$ satisfies the $h c c$. This finishes the proof of the theorem. 
Theorem 4.6 (An Analog of Dixmier's Problem 6 for Algebras that satisfy the Commutative Centralizer Condition) Let $B=\cup_{i \geq 0} B_{i}$ be a filtered algebra over an algebraically closed field of characteristic zero such that the associated graded algebra gr $B$ is a commutative domain, and $B$ satisfies the commutative centralizer condition and admits the Dixmier partition. Let $f(t) \in K[t]$ be an arbitrary polynomial of degree $>1$.

1. Let $i=1$,2. If $a \in \Delta_{i}(B)$ then $f(a) \in \Delta_{i}(B)$ provided $f(a) \notin Z(B)$.

2. Let $i=3$, 4. If $a \in \Delta_{i}(B)$ then $f(a) \in \Delta_{5}(B)$.

3. If $a \in \Delta_{5}(B)$ then $f(a) \in \Delta_{5}(B)$.

Proof. By Corollary 2.7, $a \notin Z(B)$ and $f(a) \in Z(B)$ imply $a \in B_{0}$. Clearly $B_{0} \subseteq$ $Z(B) \cup \Delta_{1,2}(B)$ since $\operatorname{gr}(B)$ is a commutative algebra.

Let $i=1,2$. By Proposition 4.5, $a \in \Delta_{i}(B) \Leftrightarrow f(a) \in \Delta_{i}(B)$ provided $f(a) \notin Z(B)$.

For $i=3,4, a \notin B_{0}$, hence $f(a) \notin Z(B), f(a) \notin \Delta_{3,4}(B)$, by Theorem 1.1, and $f(a) \notin \Delta_{1,2}(B)$, by the argument above. Therefore, $f(a) \in \Delta_{5}(B)$.

For $i=5, a \notin B_{0}$, hence $f(a) \notin Z(B), f(a) \notin \Delta_{3,4}(B)$ (Theorem 1.1), and $f(a) \notin$ $\Delta_{1,2}(B)$, hence $f(a) \in \Delta_{5}(B)$.

\section{The Weyl Division Ring satisfies the Commutative Centralizer Condition}

Let $L$ be a field, and let $\sigma$ be an automorphism of the field $L$. The skew Laurent series algebra $\mathcal{L}=L\left[\left[X, X^{-1} ; \sigma\right]\right]$ consists of (inverse) Laurent series $a=\sum_{i=-\infty}^{n} a_{i} X^{i}$ with $n \in \mathbb{Z}$ and coefficients $a_{i} \in L$. Addition and multiplication in $\mathcal{L}$ are given by the formulae:

$$
a+b=\sum\left(a_{i}+b_{i}\right) X^{i} \text { and } a b=\sum_{i}\left(\sum_{j+k=i} a_{j} \sigma^{j}\left(b_{k}\right)\right) X^{i},
$$

where $b=\sum b_{i} X^{i}$. The ring $\mathcal{L}$ is a division ring. If $a \neq 0$ then the degree $\operatorname{deg} a$ of $a$ is the maximal integer $n$ such that $a_{n} \neq 0$, and so $a=a_{n} t^{n}+\cdots$ where $a_{n} t^{n}$ (resp. $a_{n}$ ) is the leading term (resp. the leading coefficient) of $a$, and three dots denote terms of smaller degree. We define $\operatorname{deg}(0)=-\infty$. For $a=a_{n} X^{n}+\cdots, b=$ $b_{m} X^{m}+\cdots \in \mathcal{L}, a b=a_{n} \sigma^{n}\left(b_{m}\right) X^{n+m}+\cdots$, and so $\operatorname{deg}(a b)=\operatorname{deg}(a)+\operatorname{deg}(b)$ and $\operatorname{deg}(a+b) \leq \max \{\operatorname{deg}(a), \operatorname{deg}(b)\}$.

The subring $L\left[X, X^{-1} ; \sigma\right]$ of $\mathcal{L}$ that consists of all finite sums $\sum a_{i} X^{i}$ is called the skew Laurent polynomial ring. The ring $\mathcal{L}$ is a completion of $L\left[X, X^{-1} ; \sigma\right]$ with respect to the valuation $v(a):=-\operatorname{deg} a$.

The subring $\mathcal{L}_{-}:=\{a \in \mathcal{L} \mid \operatorname{deg} a \leq 0\}$ of $\mathcal{L}$ is a skew series algebra $L\left[\left[X^{-1} ; \sigma^{-1}\right]\right]$. It is a local ring with (unique) maximal ideal $\mathcal{L}_{-} X^{-1}$. The residue ring $\mathcal{L}_{-} / \mathcal{L}_{-} X^{-1}$ is canonically isomorphic to the field $L$. A nonzero element of $\mathcal{L}_{-}$is a unit iff it has degree 0 .

For a field extension $E$ of $F$ we denote by $[E: F]$ the dimension of $E$ over $F$. Let $L^{\sigma}:=\{l \in L \mid \sigma(l)=l\}$ be the subfield of $L$ of fixed elements for the automorphism $\sigma$ of the field $L$. 
Theorem 5.1 The skew Laurent series ring $\mathcal{L}=L\left[\left[X, X^{-1} ; \sigma\right]\right]$ with coefficients from a field $L$ satisfies the commutative centralizer condition provided $\sigma \neq i d_{L}$ and, for each $l \in L \backslash L^{\sigma}, \sigma^{i}(l) \neq l$ for all $i \geq 1$.

1. If $u \in L \backslash L^{\sigma}$, then $C(u)=L$.

2. If $u \in \mathcal{L} \backslash L, \operatorname{deg} u=0$, and the leading coefficient $u_{0}$ of $u$ does not belong to $L^{\sigma}$, then $C(u) \subseteq \mathcal{L}_{-}$, and the map $C(u) \rightarrow L=\mathcal{L}_{-} / \mathcal{L}_{-} X^{-1}, c \rightarrow c+\mathcal{L}_{-} X^{-1}$, is a $L^{\sigma}$-algebra isomorphism, $\left[C(u): L^{\sigma}(u)\right]=\left[L: L^{\sigma}\left(u_{0}\right)\right]$.

3. If $u \in \mathcal{L} \backslash L$ with $\operatorname{deg} u \neq 0$ then $C(u)=L^{\sigma}\left[\left[v, v^{-1}\right]\right]$ is a Laurent series field with coefficients from the field $L^{\sigma}$ where $v$ is an element of $C(u)$ which has the least positive degree. $\left[C(u): L^{\sigma}\left[\left[u, u^{-1}\right]\right]\right]=\left|\frac{\operatorname{deg} u}{\operatorname{deg} v}\right|<\infty$.

Proof. Observe that the centralizer $C=C(u)$ of any nonzero element of $\mathcal{L}$ is a skew subfield of $\mathcal{L}$.

1. By the assumption $u \notin L^{\sigma}=L^{\sigma^{i}}$ (for all $0 \neq i \in \mathbb{Z}$ ), which means that $\sigma^{i}(u)-u \neq 0$ for all $0 \neq i \in \mathbb{Z}$. Now, $a=\sum a_{i} X^{i} \in C(u)$ iff $0=a u-u a=\sum a_{i}\left(\sigma^{i}(u)-u\right) X^{i}$ iff $a_{i}=0$ for all $0 \neq i \in \mathbb{Z}$ iff $C(u)=L$.

2. By the assumption, the element $u$ has the form $\sum_{j \leq 0} u_{j} X^{j}$ with $u_{0} \in L \backslash L^{\sigma}$. Let $0 \neq a=\sum a_{i} X^{i} \in C(u)$. Then the leading term of $a$, say $a_{d} X^{d}$, commutes with $u_{0}$, hence $d=0$ since $u_{0} \notin L^{\sigma^{i}}=L^{\sigma}$ for all $0 \neq i \in \mathbb{Z}$. Thus $a=\sum_{i \leq 0} a_{i} X^{i}$ and $C \subseteq \mathcal{L}_{-}$. Now,

$$
0=a u-u a=\sum_{s \leq 0}\left(\sum_{i+j=s}\left(a_{i} \sigma^{i}\left(u_{j}\right)-u_{j} \sigma^{j}\left(a_{i}\right)\right)\right) X^{s} .
$$

Equating the coefficients of $X^{s}$ to 0 in the equality above we obtain the infinite system of equations with unknowns $a_{i}, i \leq 0$,

$$
\left(\sigma^{s}\left(u_{0}\right)-u_{0}\right) a_{s}+\sum_{i+j=s, i>s}\left(a_{i} \sigma^{i}\left(u_{j}\right)-u_{j} \sigma^{j}\left(a_{i}\right)\right)=0, s \leq 0 .
$$

For a given $a_{0}$, the system has a unique solution since $\sigma^{s}\left(u_{0}\right)-u_{0} \neq 0$, for all $s<0$ (since $\left.u_{0} \notin L^{\sigma}\right)$. So, the map $C(u) \rightarrow K(H), c \rightarrow c+\mathcal{L}_{-} X^{-1}$, is a $L^{\sigma}$-algebra isomorphism, hence $\left[C(u): L^{\sigma}(u)\right]=\left[L: L^{\sigma}\left(u_{0}\right)\right]$.

3. Since $C(u)=C\left(u^{-1}\right)$ we may assume that $d:=\operatorname{deg} u>0$. Then the set $G:=$ $\{\operatorname{deg} c \mid 0 \neq c \in C(u)\}$ is a nonzero subgroup of $\mathbb{Z}$ that contains $\mathbb{Z} d$, hence $G=\mathbb{Z} t$ where an integer $t$ is the least positive element of $G$. Clearly, $t \mid d$. Fix an element $v \in C(u)$ with $\operatorname{deg} v=t$. We claim that $C(u)=L^{\sigma}\left[\left[v, v^{-1}\right]\right]$. Obviously, $L^{\sigma}\left[\left[v, v^{-1}\right]\right] \subseteq C(u)$. In order to prove the reverse inclusion, we first show that, for a given $\operatorname{deg} c$, the leading term of $c \in C(u)$ is unique up to a factor of $L^{\sigma}$.

So, let elements $c, c^{\prime} \in C(u)$ have the same degree, say $n$, and the leading terms $\alpha X^{n}$ and $\alpha^{\prime} X^{n}$ respectively. These leading terms commute with the leading term, say $u_{d} X^{d}$, of the element $u$ :

$0=\left[\alpha X^{n}, u_{d} X^{d}\right]=\left(\alpha \sigma^{n}\left(u_{d}\right)-u_{d} \sigma^{d}(\alpha)\right) X^{n+d}$ and $0=\left[\alpha^{\prime} X^{n}, u_{d} X^{d}\right]=\left(\alpha^{\prime} \sigma^{n}\left(u_{d}\right)-u_{d} \sigma^{d}\left(\alpha^{\prime}\right)\right) X^{n+d}$, 
and so $\frac{\sigma^{d}(\alpha)}{\alpha}=\frac{\sigma^{n}\left(u_{d}\right)}{u_{d}}=\frac{\sigma^{d}\left(\alpha^{\prime}\right)}{\alpha^{\prime}}$ which implies that $\sigma^{d}\left(\frac{\alpha}{\alpha^{\prime}}\right)=\frac{\alpha}{\alpha^{\prime}}$, hence $\frac{\alpha}{\alpha^{\prime}} \in L^{\sigma^{d}}=L^{\sigma}$, as required.

Let $a$ be an arbitrary element of $C(u)$. The elements $a$ and $v^{i_{1}}, i_{1}:=t^{-1} \operatorname{deg} a$, of $C(u)$ have the same degree $\operatorname{deg} a$, so we can choose a scalar, say $\lambda_{1} \in L^{\sigma}$, such that the elements $a$ and $\lambda_{1} v^{i_{1}}$ have the same leading term. Hence the element $a_{1}:=a-\lambda_{1} v^{i_{1}} \in C(u)$ has degree $\operatorname{deg} a_{1}<\operatorname{deg} a$. Repeating the same argument for the element $a_{1} \in C(u)$, we can find a scalar $\lambda_{2} \in L^{\sigma}$ and an integer $i_{2} \in \mathbb{Z}$ such that the element $a_{2}:=a-\lambda_{2} v^{i_{2}} \in C(u)$ has degree $\operatorname{deg} a_{2}<\operatorname{deg} a_{1}$. Proceeding in this way, we can find infinitely many elements of $C(u)$,

$$
a_{\nu}:=a_{\nu-1}-\lambda_{\nu} v^{i_{\nu}}, \lambda_{\nu} \in L^{\sigma}, \nu \geq 1
$$

such that $\operatorname{deg} a_{1}>\operatorname{deg} a_{2}>\cdots$ and $i_{1}>i_{2}>\cdots$. So,

$$
a=\sum_{\nu \geq 1} \lambda_{\nu} v^{i_{\nu}} \in L^{\sigma}\left[\left[V, V^{-1}\right]\right]
$$

thus $C(u) \subseteq L^{\sigma}\left[\left[V, V^{-1}\right]\right]$, as required. A degree argument shows that $\left[C(u):, L^{\sigma}\left[\left[u, u^{-1}\right]\right]\right]=$ $\left|\frac{\operatorname{deg} u}{\operatorname{deg} v}\right|<\infty$.

Corollary 5.2 Let $\mathcal{L}$ be as in Theorem 5.1. Then the centre $Z(\mathcal{L})=L^{\sigma}$.

Proof. By Theorem [5.1] $(1,3), C(X)=L^{\sigma}\left[\left[X, X^{-1}\right]\right]$ and $C(u)=L$ for each $u \in L \backslash L^{\sigma}$. Now,

$$
L^{\sigma} \subseteq Z(\mathcal{L}) \subseteq C(u) \cap C(X)=L \cap L^{\sigma}\left[\left[X, X^{-1}\right]\right]=L^{\sigma},
$$

and so $Z(\mathcal{L})=L^{\sigma}$.

Let $K(H)$ and $K(H, C)$ be fields of rational functions with coefficients from the field $K$ of characteristic zero in one and two variables. It can be easily verified that the following four division rings satisfy the conditions of Theorem [5.1, and so satisfy the commutative centralizer condition.

1. $\mathcal{A}:=K(H)\left[\left[X, X^{-1} ; \sigma\right]\right], \sigma \in \operatorname{Aut}_{K}(K(H)), \sigma(H)=H-1$.

2. $\mathcal{B}_{\lambda}:=K(H)\left[\left[X, X^{-1} ; \tau\right]\right], \tau \in \operatorname{Aut}_{K}(K(H)), \tau(H)=\lambda H$ where $\lambda \in K^{*}$ is not an $i^{\text {th }}$ root of 1 for all $i \geq 1$.

3. $\mathcal{C}:=K(H, C)\left[\left[X, X^{-1} ; \sigma\right]\right], \sigma \in \operatorname{Aut}_{K}(K(H, C)), \sigma(H)=H-1, \sigma(C)=C$.

4. $\mathcal{E}_{\lambda}:=K(H, C)\left[\left[X, X^{-1} ; \tau\right]\right], \tau \in \operatorname{Aut}_{K}(K(H, C)), \tau(H)=\lambda H, \tau(C)=C$, where $\lambda \in K^{*}$ is not an $i^{\text {th }}$ root of 1 for all $i \geq 1$.

By Corollary 5.2 ,

$$
Z(\mathcal{A})=K, Z\left(\mathcal{B}_{\lambda}\right)=K, Z(\mathcal{C})=K(C), Z\left(\mathcal{E}_{\lambda}\right)=K(C) .
$$

Next we show that certain algebras (considered in Introduction) are subalgebras of one of these division rings, and then applying Theorem 1.3 we prove that they admit 
the Dixmier partition and satisfy both the homogeneous centralizer condition and the commutative centralizer condition.

The Weyl algebra $A_{1}$ can be identified with its image in the division $\operatorname{ring} \mathcal{A}$ via the $K$-algebra monomorphism $A_{1} \rightarrow \mathcal{A}, x \rightarrow X, \partial \rightarrow H X^{-1}$, as follows from the commutator $\left(H X^{-1}\right) X-X\left(H X^{-1}\right)=H-(H-1) X X^{-1}=1$. The Weyl algebra $A_{1}$ is a Noetherian domain, its division ring $Q\left(A_{1}\right)$ (so-called, the Weyl division ring) is a subring of $\mathcal{A}$ by the universality of localization, and so the Weyl division ring $Q\left(A_{1}\right)$ satisfies the $c c c$.

Clearly, the Weyl division ring $Q\left(A_{1}\right)$ has the trivial centre

$$
Z\left(Q\left(A_{1}\right)\right)=K
$$

since (by Theorem [5.1) $C(X, \mathcal{A})=K\left[\left[X, X^{-1}\right]\right], C(H=\partial x, \mathcal{A})=K(H)$, and so

$$
\begin{aligned}
K & \subseteq Z\left(Q\left(A_{1}\right)\right) \subseteq C\left(H, Q\left(A_{1}\right)\right) \cap C\left(X, Q\left(A_{1}\right)\right)=Q\left(A_{1}\right) \cap C(H, \mathcal{A}) \cap C(X, \mathcal{A}) \\
& =Q\left(A_{1}\right) \cap K(H) \cap K\left[\left[X, X^{-1}\right]\right]=Q\left(A_{1}\right) \cap K=K .
\end{aligned}
$$

Given a nonzero polynomial $a=a(H) \in K[H]$. The algebra

$$
A(a):=K\langle X, Y, H \mid X H=(H-1) X, Y H=(H+1) Y, Y X=a(H), X Y=a(H-1)\rangle
$$

is called the noncommutative deformation of type-A Kleinian singularities [5], [13, 9]. The algebra $A(a)$ is a subalgebra of the division ring $\mathcal{A}$ via the $K$-algebra monomorphism $A(a) \rightarrow \mathcal{A}, X \rightarrow X, Y \rightarrow a X^{-1}, H \rightarrow H$. Note that all prime infinite dimensional factor algebra $B_{\lambda}:=U \operatorname{sl}(2) /(C-\lambda) \simeq A(\lambda-H(H+1))$ are of this type where $\lambda \in K$ and $C$ is the Casimir element of $U \operatorname{sl}(2)$ (see Section [6).

The Gelfand-Kirillov dimension $\mathrm{GK}\left(A_{1}\right)=\mathrm{GK}(A(a))=2$. So, the next result follows from Theorem 1.3, and is a generalization to the Weyl division ring of the result of Amitsur that the centralizer of an arbitrary non-scalar element is a commutative algebra [1].

Corollary 5.3 1. The Weyl division ring $Q\left(A_{1}\right)$ ) satisfies the commutative centralizer condition.

2. The first Weyl algebra $A_{1}$, the noncommutative deformations of the type-A Kleinian singularities, and any noncommutative subalgebra of the Weyl division ring $Q\left(A_{1}\right)$ with Gelfand-Kirillov dimension $<3$ admits the Dixmier partition and satisfy both the homogeneous centralizer condition and the commutative centralizer condition.

The quantum plane $\Lambda=K\langle x, y \mid x y=\lambda y x\rangle$, where $\lambda \in K^{*}$ is not an $i^{\text {th }}$ root of 1 for all $i \geq 1$, can be identified with its image in the division ring $\mathcal{B}_{\lambda}$ under the $K$-algebra monomorphism $\Lambda \rightarrow \mathcal{B}_{\lambda}, x \rightarrow X, y \rightarrow H X^{-1}$, as follows from $X\left(H X^{-1}\right)=\lambda\left(H X^{-1}\right) X$. The quantum plane $\Lambda$ is a Noetherian domain of Gelfand-Kirillov dimension 2, its division ring $Q(\Lambda)$ is a subring of $\mathcal{B}_{\lambda}$ by the universality of localization, and so the division ring $Q(\Lambda)$ satisfies the $c c c$. 
The quantum Weyl algebra $A_{1}(\mu):=K\langle x, \partial \mid \partial x-\mu x \partial=1\rangle, 1 \neq \mu \in K^{*}$, is a subalgebra of the division ring $\mathcal{B}_{\mu}$ via the $K$-algebra monomorphism $A_{1}(\mu) \rightarrow \mathcal{B}_{\mu}, \partial \rightarrow X, x \rightarrow$ $(\mu-1)^{-1}(Y-1) X^{-1}$, as follows from

$$
X(\mu-1)^{-1}(Y-1) X^{-1}-\mu(\mu-1)^{-1}(Y-1) X^{-1} X=(\mu-1)^{-1}(\mu Y-1-\mu(Y-1))=1 .
$$

The quantum Weyl algebra $A_{1}(\mu)$ is a Noetherian domain of Gelfand-Kirillov dimension 2 , its division ring $Q\left(A_{1}(\mu)\right)$ is a subalgebra of $\mathcal{B}_{\mu}$, and so satisfies the ccc.

Corollary 5.4 1. The division rings of the quantum plane and the quantum Weyl algebra satisfy the commutative centralizer condition.

2. The quantum plane $\Lambda$, the quantum Weyl algebra $A_{1}(\mu)$, and any noncommutative subalgebra of the division ring $Q(\Lambda)$ with Gelfand-Kirillov dimension $<3$ admits the Dixmier partition and satisfy both the homogeneous centralizer condition and the commutative centralizer condition.

\section{The Algebras $U s l(2)$ and $U_{q} s l(2)$ Admit the Dixmier Partition and Satisfy the $c c c$ and $h c c$}

In this section we prove that the universal enveloping algebra $U \operatorname{sl}(2)$ of the Lie algebra $s l(2)$ and its quantum analog $U_{q} s l(2)$ admit the Dixmier partition and satisfy the $c c c$ and $h c c$ (Propositions 6.1 and 6.2)). We can not apply directly Theorem 1.3 since these algebras have Gelfand-Kirillov dimension 3, but modifying arguments slightly the proof of the results for the algebras $U s l(2)$ and $U_{q} s l(2)$ proceeds along the line of the proof of Theorem 1.3.

The universal enveloping algebra

$$
U \operatorname{sl}(2)=K\langle X, Y, H \mid[H, X]=X,[H, Y]=-Y,[X, Y]=2 H\rangle
$$

of the Lie algebra $\operatorname{sl}(2)$ is a $K$-subalgebra of the division ring $\mathcal{C}$ via the $K$-algebra monomorphism

$$
U \operatorname{sl}(2) \rightarrow \mathcal{C}, X \rightarrow X, Y \rightarrow(C-H(H+1)) X^{-1}, H \rightarrow H,
$$

where $C:=Y X+H(H+1)$ is the Casimir element of the algebra $U s l(2)$, an algebra generator for the centre $Z(U s l(2))=K[C]$ of the algebra $U s l(2)$.

The universal enveloping algebra $U s l(2)$ is a Noetherian domain of Gelfand-Kirillov dimension 3, so its division ring $Q(U s l(2))$ is a subalgebra of the division $\operatorname{ring} \mathcal{C}$, and so both algebras $U s l(2)$ and $Q(U s l(2))$ satisfy the $c c c$. The centre $Z(Q(U s l(2)))=K(C)$, the field of rational functions, as follows from (using Theorem 5.11)

$K(C) \subseteq Z(Q(U \operatorname{sl}(2))) \subseteq \mathcal{C} \cap C(H, \mathcal{C}) \cap C(X, \mathcal{C})=\mathcal{C} \cap K(H, C) \cap K(C)\left[\left[X, X^{-1}\right]\right]=K(C)$

So, we have proved the first statement of the next result. 
Proposition 6.1 $\quad$ 1. The universal enveloping algebra $U$ sl $(2)$ and its division ring $Q(U s l(2))$ satisfy the commutative centralizer condition, and the centre $Z(Q(U \operatorname{sl}(2)))=K(C)$.

2. The universal enveloping algebra Usl(2), or any noncommutative subalgebra $A$ of the division ring $Q(U \operatorname{sl}(2))$ with Gelfand-Kirillov dimension $\mathrm{GK}(A)<4$ and such that $A \cap K(C) \neq K$ admits the Dixmier partition and satisfies both the homogeneous centralizer condition and the commutative centralizer condition.

Proof. 2. We split the proof into several steps.

Step 1. $\operatorname{GK}(C(a, A)) \geq 2$ for all $a \in A \backslash Z(A)$.

In order to prove this fact, let us fix an element, say $z$, of the set $(A \cap K(C)) \backslash K$. Then the polynomial algebra $K[z]$ is the subalgebra of the centre $Z(A)$. Let $a \in A \backslash Z(A)$. The centralizer $C(a, A)$ is a commutative algebra (since the division ring $Q(U \operatorname{sl}(2))$ satisfies the $c c c$ ) that contains the elements $a$ and $z$. It suffices to show that the elements $a$ and $z$ are algebraically independent.

If $\operatorname{deg} a \neq 0($ in $\mathcal{C}$ ) then this fact is evident since $\operatorname{deg} z=0$.

If $a \in K(H, C)$ then $a \notin K(C)$ (otherwise $a \in A \cap K(C)=A \cap Z(U s l(2)) \subseteq Z(A)$, a contradiction), and so $a$ is not an algebraic elements over the field $K(C)$, hence $a$ and $z$ are algebraically independent.

In the remaining case when $\operatorname{deg} a=0, a \notin K(H, C)$, suppose that the elements $a$ and $z$ are algebraically dependent. This implies that $a$ is an algebraic element over the field $K(C)$ since $z \in K(C)$. Then the leading term, say $a_{0} \in K(H, C)$, of $a$ is algebraic over the field $K(C)$, and so $a_{0} \in K(C)$. Then the element $a-a_{0}$ is algebraic over $K(C)$ which is impossible since $\operatorname{deg}\left(a-a_{0}\right)<0$. This contradiction proves Step 1 .

Step 2. The algebra $A$ admits the Dixmier partition.

The algebra $\overline{\mathcal{C}}=\bar{K}(H, C)\left[\left[X, X^{-1} ; \sigma\right]\right]$ is a domain, so is $\bar{A}$ since $\bar{A} \subseteq \overline{\mathcal{C}}$. So, without loss of generality we may assume that $K=\bar{K}$. Suppose that the algebra $A$ does not admit the Dixmier partition. Then there exists an element $a \in A \backslash Z(A)$ such that $N(a) \neq C(a)$ and $D(a) \neq C(a)$. Let us fix nonzero elements $x \in N(a, 1, A) \backslash C(a)$ and $y \in D(a, \lambda, A)$ for some $0 \neq \lambda \in K$. Then, for all $i, j \geq 0$,

$$
C(a)^{*} x^{i} y^{j} \subseteq F(a, j \lambda, i, A) \backslash F(a, j \lambda, i-1, A)
$$

where $C(a)^{*}:=C(a) \backslash\{0\}$, and so the torsion algebra

$$
F(a) \supseteq \sum_{i, j \geq 0} C(a) x^{i} y^{j}=\bigoplus_{i, j \geq 0} C(a) x^{i} y^{j} \supseteq \bigoplus_{i, j \geq 0} K[a, z] x^{i} y^{j}
$$

hence $\operatorname{GK}(F(a)) \geq 4$ which contradicts to the fact that $\operatorname{GK}(F(a)) \leq \operatorname{GK}(A)<4$. This proves Step 2.

Step 3. The algebra A satisfies the hcc.

We still may assume that $K=\bar{K}$. Let $a \in A \backslash Z(A)$, and let $C=C(a)$. Suppose that the algebra $C$ contains a strongly nilpotent element (resp. a weakly nilpotent element). Then, by Proposition 4.5, all the elements of the set $C \backslash Z(A)$ are strongly nilpotent (resp. weakly nilpotent). 
Suppose that the algebra $C$ does not contain a nilpotent element. Then either all the elements of the set $C \backslash Z(A)$ are generic or, otherwise, there exists a semi-simple element, say $a \in C \backslash Z(A)$. Suppose that $b$ is another semi-simple element of $C$. Then $a b=b a$ since $C$ is a commutative algebra, $[a, p]=\lambda p$, and $[b, q]=\mu q$ for some nonzero elements $p, q \in A$ and some nonzero scalars $\lambda, \mu \in K^{*}$. Then, for some $i \geq 1$, the element $p^{i}$ is an eigenvector for the inner derivation ad $b$ of the algebra $A$ since otherwise, by Proposition 4.4 the sum $M=\sum_{i, j \geq 0} C q^{i} p^{j}$ is a free left $C$-submodule of $A$ with the free basis $\left\{q^{i} p^{j} \mid i, j \geq 0\right\}$. By Step 1, $C$ contains the polynomial algebra $K[a, z]$, and so $A \supseteq \oplus_{i, j, k, l \geq 0} K a^{k} z^{l} q^{i} p^{j}$. Therefore the subalgebra $\Lambda$ of $A$ generated by the elements $a, z, p$, and $q$ has GelfandKirillov dimension $\geq 4$, which contradicts to the fact that $\operatorname{GK}(\Lambda) \leq \operatorname{GK}(A)<4$. So, we have proved that, for some $i \geq 1, p^{i}$ is a common eigenvector for the inner derivations ad $a$ and $\operatorname{ad} b: \quad\left[a, p^{i}\right]=i \lambda p^{i}$ and $\left[b, p^{i}\right]=\gamma p^{i}$ for some $\gamma \in K$. We must have $\gamma \neq 0$ since otherwise the non-central elements $b$ and $p^{i}$ commute, and so they have the common centralizer $C(b)=C\left(p^{i}\right)$. By the assumption, the non-central elements $a$ and $b$ commute, so $C(a)=C(b)$ which implies that the elements $a$ and $p^{i}$ commute which is impossible. Now, by Lemma 4.3. $b \in K^{*} a+Z(A)$, hence $K^{*} a+Z(A)$ is the set of all semi-simple elements of $C$. This finishes the proof of Step 3 and the proposition.

Suppose that a scalar $q \in K^{*}$ is not a root of 1 , and let $h:=q-q^{-1}$. The quantum

$$
U_{q} s l(2)=K\left\langle X, Y, H, H^{-1} \mid X H=q H X, Y H=q^{-1} H Y,[X, Y]=h^{-1}\left(H^{2}-H^{-2}\right)\right\rangle
$$

is a $K$-subalgebra of the division ring $\mathcal{E}_{q}$ via the $K$-algebra monomorphism

$$
U_{q} \operatorname{sl}(2) \rightarrow \mathcal{E}_{q}, X \rightarrow X, Y \rightarrow\left(C+(2 h)^{-1}\left(\frac{H^{2}}{q^{2}-1}-\frac{H^{-2}}{q^{-2}-1}\right)\right) X^{-1}, H \rightarrow H,
$$

where $C:=Y X-(2 h)^{-1}\left(\frac{H^{2}}{q^{2}-1}-\frac{H^{-2}}{q^{-2}-1}\right)$ is the (quantum) Casimir element of the algebra $U_{q} s l(2)$, an algebra generator for the centre $Z\left(U_{q} s l(2)\right)=K[C]$ of the algebra $U_{q} s l(2)$.

The algebra $U_{q} s l(2)$ is a Noetherian domain of Gelfand-Kirillov dimension 3, so its division ring $Q\left(U_{q} s l(2)\right)$ is a subalgebra of the division ring $\mathcal{E}_{q}$ which implies that both algebra $U_{q} s l(2)$ and $Q\left(U_{q} s l(2)\right)$ satisfy the $c c c$. The centre $Z\left(Q\left(U_{q} s l(2)\right)\right)=K(C)$ since

$K(C) \subseteq Z\left(Q\left(U_{q} s l(2)\right)\right) \subseteq \mathcal{E}_{q} \cap C\left(H, \mathcal{E}_{q}\right) \cap C\left(X, \mathcal{E}_{q}\right)=\mathcal{E}_{q} \cap K(H, C) \cap K(C)\left[\left[X, X^{-1}\right]\right]=K(C)$.

So, we have proved the first statement of the next result.

Proposition 6.2 1. The quantum $U_{q} s l(2)$ and its division ring $Q\left(U s_{q} l(2)\right)$ satisfy the commutative centralizer condition, and the centre $Z\left(Q\left(U_{q} s l(2)\right)\right)=K(C)$.

2. The quantum $U_{q} s l(2)$, or any noncommutative subalgebra $A$ of the division ring $Q\left(U_{q} s l(2)\right)$ with Gelfand-Kirillov dimension $\mathrm{GK}(A)<4$ and such that $A \cap K(C) \neq K$ admits the Dixmier partition and satisfies both the homogeneous centralizer condition and the commutative centralizer condition. 
Proof. 2. The proof is literally the same as the proof to Proposition 6.1 and we leave it for the reader.

We left it for the reader to prove that (using a proper embedding into the division ring of the type $\mathcal{E}_{q^{\prime}}$ ) the quantum Heisenberg algebra [17], [18]:

$$
\mathcal{H}_{q}=K\left\langle X, Y, H \mid X H=q^{2} H X, Y H=q^{-2} H Y, X Y-q^{-2} Y X=q^{-1} H\right\rangle
$$

and the Witten's first deformation:

$$
E=K\left\langle E_{0}, E_{-}, E_{+} \mid\left[E_{0}, E_{+}\right]_{q}=E_{+},\left[E_{-}, E_{0}\right]_{q}=E_{-},\left[E_{+}, E_{-}\right]=E_{0}-(q-1 / q) E_{0}^{2}\right\rangle
$$

admit the Dixmier partition and satisfy both the homogeneous centralizer condition and the commutative centralizer condition (where $q \in K^{*}$ is not a root of $1,[a, b]_{q}:=q a b-q^{-1} b a$ ).

\section{$7 \quad$ The Ring $\mathcal{D}(X)$ of Differential Operators on a Smooth Irreducible Algebraic Curve $X$}

In this section, let $K$ be an algebraically closed field of characteristic zero, let $X$ be a smooth irreducible algebraic curve over $K$. The coordinate algebra $\mathcal{O}=\mathcal{O}(X)$ on $X$ is a finitely generated regular domain of Krull dimension 1 . We denote by $Q=Q(X)$ its quotient field. Let $\mathcal{D}(X)=\mathcal{D}(\mathcal{O}(X))$ be the ring of differential operators on $X$. Recall the definition and basic properties of the algebra $\mathcal{D}(\mathcal{O})$ (see [21], Ch. 15, for details).

A ring of (K-linear) differential operators $\mathcal{D}(\mathcal{O})=\cup_{i \geq 0} \mathcal{D}(\mathcal{O})_{i}$ on $X$ is a subalgebra of $\operatorname{End}_{K}(\mathcal{O})$ where $\mathcal{D}(\mathcal{O})_{0}=\left\{u \in \operatorname{End}_{K}(\mathcal{O}): u r-r u=0\right.$, for all $\left.r \in \mathcal{O}\right\}=\operatorname{End}_{\mathcal{O}}(\mathcal{O}) \simeq \mathcal{O}$,

$$
\mathcal{D}(\mathcal{O})_{i}=\left\{u \in \operatorname{End}_{K}(\mathcal{O}): u r-r u \in \mathcal{D}(\mathcal{O})_{i-1}, \text { for all } r \in \mathcal{O}\right\}
$$

$\mathcal{D}(\mathcal{O})_{i} \mathcal{D}(\mathcal{O})_{j} \subseteq \mathcal{D}(\mathcal{O})_{i+j}$ for all $i, j \geq 0$, so $\mathcal{D}(\mathcal{O})$ is a positively filtered algebra. We say that an element $u \in \mathcal{D}(\mathcal{O})_{i} \backslash \mathcal{D}(\mathcal{O})_{i-1}$ has order $i$ denoted ord $(u)$. The subalgebra $\Delta(\mathcal{O})$ of $\mathcal{D}(\mathcal{O})$ generated by the coordinate algebra $\mathcal{O} \equiv \operatorname{End}_{\mathcal{O}}(\mathcal{O})$ and the set $\operatorname{Der}_{K}(\mathcal{O})$ of all $K$-derivations of the algebra $\mathcal{O}$ is called the derivation ring of $\mathcal{O}$.

- $\operatorname{Der}_{K}(\mathcal{O})$ is a finitely generated projective $\mathcal{O}$-module of rank 1.

- $\mathcal{D}(\mathcal{O})=\Delta(\mathcal{O})$.

- $\mathcal{D}(\mathcal{O})$ is a central simple (left and right) Noetherian domain of Gelfand-Kirillov dimension 2.

- If $S$ is a multiplicatively closed subset of $\mathcal{O}$ then $S$ is a (left and right) Ore set of $\mathcal{D}(\mathcal{O})$, and $\mathcal{D}\left(S^{-1} \mathcal{O}\right)=S^{-1} \mathcal{D}(\mathcal{O})$.

- The associative graded algebra gr $\mathcal{D}(\mathcal{O})=\bigoplus_{i \geq 0} \mathcal{D}(\mathcal{O})_{i} / \mathcal{D}(\mathcal{O})_{i-1}$ is a commutative domain, and so $\operatorname{ord}(u v)=\operatorname{ord}(u)+\operatorname{ord}(v)$ for nonzero elements $u, v \in \mathcal{D}(\mathcal{O})$. 
- If $\mathcal{O}=K[x]$ is a polynomial algebra then $\mathcal{D}(K[x])=A_{1}$ is the Weyl algebra.

- Let $\mathbf{m}$ be a maximal ideal of $\mathcal{O}$. Then there exists an element $c=c(\mathbf{m}) \in \mathcal{O} \backslash \mathbf{m}$ such that the localization $\mathcal{D}(\mathcal{O})_{c}$ of $\mathcal{D}(\mathcal{O})$ at the powers of the element $c$ is an Ore extension $\mathcal{O}_{c}[t ; \delta]$ of $\mathcal{O}_{c}$, where $\delta \in \operatorname{Der}_{K}\left(\mathcal{O}_{c}\right)$, and $\delta=\frac{d}{d x}$ where $x \in Q(X)$ is a transcendence basis for the field $Q(X)$. Clearly, $\operatorname{ker} \delta=K$ in $Q(X)$ (since $K=\bar{K}$ ).

Let $R$ be a ring, and let $\delta$ be a derivation of the ring $R$. The skew polynomial ring (or the Ore extension) $T=R[t ; \delta]$ is a ring generated freely over $R$ by an element $t$ subject to the defining relation $t r=r t+\delta(r)$ for all $r \in R$. An element $a$ of $T$ is a unique sum $\sum a_{i} t^{i}$ where $a_{i} \in R . \operatorname{deg}(a)=\max \left\{i \mid a_{i} \neq 0\right\}$ is called the degree of $a, \operatorname{deg}(0):=-\infty$. A nonzero element $a$ of degree $n$ can be written as $a_{n} t^{n}+\cdots$ where by three dots we denote the smaller terms, $a_{n} t^{n}$ and $a_{n}$ are called the leading term and the leading coefficient of $a$ respectively. If $R$ is a domain then $\operatorname{deg}(a b)=\operatorname{deg}(a)+\operatorname{deg}(b)$ for all $a, b \in T$, and so $T$ is a domain.

The algebra $\mathcal{D}(X)$ is a Noetherian domain, so its (left and right) quotient ring $\Gamma(X)$ is a division ring. We fix the element $c$. Then we have the chain of algebras

$$
\mathcal{D}(X) \subseteq \mathcal{D}(X)_{c}=\mathcal{O}_{c}[t ; \delta] \subseteq Q(X)[t ; \delta] \subseteq \Gamma(X) .
$$

Our first goal is to prove that the algebras $\mathcal{D}(X)$ and $\Gamma(X)$ satisfy the commutative centralizer condition (Corollary [7.2). We use the same strategy as before: we embed $\Gamma(X)$ into a bigger division ring $\mathcal{R}$ that satisfies the $c c c(\mathcal{R}$ will be a completion of the Ore extension $Q(X)[t ; \delta]$ with respect to the (additive) valuation determined by the degree function).

The centre of the algebra $\mathcal{D}(X)$ is $K$, and the Gelfand-Kirillov dimension is $\operatorname{GK}(\mathcal{D}(X))=$ 2 , so, by Corollary 3.2, the ring of differential operators $\mathcal{D}(X)$ admits the Dixmier partition:

$$
\mathcal{D}(X) \backslash K=\cup_{i=1}^{5} \Delta_{i}(\mathcal{D}(X)) .
$$

By the very definition of the ring of differential operators $\mathcal{D}(X), \mathcal{O}(X) \backslash K \subseteq \Delta_{1}(\mathcal{D}(X))$.

Let $L$ be a ring, and let $\delta$ be a derivation of $L$. The formal pseudo-differential operator ring $\mathcal{R}:=L\left(\left(t^{-1} ; \delta\right)\right)$ consists of inverse Laurent series $a=\sum_{i=-\infty}^{n} a_{i} t^{i}$ with $n \in \mathbb{Z}$ and coefficients $a_{i} \in L$ where

$$
t r=r t+\delta(r) \text { for all } r \in L .
$$

If $a \neq 0$ then the degree $\operatorname{deg}(a)$ of $a$ is the maximal integer $n$ such that $a_{n} \neq 0$, and so $a=a_{n} t^{n}+\cdots$ where $a_{n} t^{n}$ (resp. $a_{n}$ ) is the leading term (resp. the leading coefficient) of $a$, and three dots denote terms of smaller degree. We define $\operatorname{deg}(0)=-\infty$. Suppose that $L$ is a domain and $a, b \in \mathcal{R}$. Then $\operatorname{deg}(a b)=\operatorname{deg}(a)+\operatorname{deg}(b)$ and $\operatorname{deg}(a+b) \leq$ $\max \{\operatorname{deg}(a), \operatorname{deg}(b)\}$. The subset $\mathcal{R}_{-}$of $\mathcal{R}$ that consists of elements degree $\leq 0$ is a subring of $\mathcal{R}$, and $\mathcal{R}_{-} t^{-1}$ is a (two-sided) ideal of $\mathcal{R}_{-}$such that $\mathcal{R}_{-} / \mathcal{R}_{-} t^{-1} \simeq L$. If $L$ is a division ring then $\mathcal{R}$ is a division ring. The skew polynomial ring $L[t ; \delta]$ is a subring of $\mathcal{R}$.

The next result is a version of the result of K. R. Goodearl [14, Theorem 3.5. 
Lemma 7.1 Let $L$ be a proper field extension of the field $K$, let $\delta$ be a derivation of the field $L$ such that $\operatorname{ker} \delta=K$. Then the formal pseudo-differential operator ring $\mathcal{R}=L\left(\left(t^{-1} ; \delta\right)\right)$ satisfies the commutative centralizer condition.

1. If $u \in L \backslash K$, then $C(u)=L$.

2. If $u \in \mathcal{R} \backslash L$, $\operatorname{deg} u=0$, and the leading coefficient $u_{0}$ of $u$ does not belong to $K$ then $C(u) \subseteq \mathcal{R}_{-}$, and the map $C(u) \rightarrow L=\mathcal{R}_{-} / \mathcal{R}_{-} t^{-1}, c \rightarrow c+\mathcal{R}_{-} t^{-1}$, is a K-algebra isomorphism, $[C(u): K(u)]=\left[L: K\left(u_{0}\right)\right]<\infty$.

3. If $u \in \mathcal{R} \backslash L$, and $\operatorname{deg} u \neq 0$, then $C(u)=K\left[\left[v, v^{-1}\right]\right]$ is a Laurent series field where $v$ is an element of $C(u)$ which has least positive degree. $\left[C(u): K\left[\left[u, u^{-1}\right]\right]\right]=\left|\frac{\operatorname{deg} u}{\operatorname{deg} v}\right|<$ $\infty$.

Proof. Note that $\mathcal{R}$ is a division ring, and so the centralizer $C=C(u, \mathcal{R})$ of any nonzero element $u$ of $\mathcal{R}$ is a division ring.

1. Clearly, $L \subseteq C$. Suppose that $L \neq C$. Then we can choose a nonzero element, say $a$, of $C$ of nonzero degree, say $n$. Let $\alpha t^{n}(\alpha \in L)$ be its leading term. The leading term of the commutator $[a, u]=0$ is equal to $n \alpha t^{n-1} \delta(u) \neq 0$ since $u \notin K=\operatorname{ker} \delta$, a contradiction. So, $C=L$.

2. By the assumption, $u=\sum_{j \leq 0} u_{j} t^{j}$ and $u_{0} \in L \backslash K$. Let $0 \neq a=\sum a_{i} t^{i} \in C$. By the previous argument, the leading term of $a$, say $a_{d} t^{d}$, belongs to $L$, thus $\operatorname{deg} a=d=0$, $a=\sum_{i \leq 0} a_{i} t^{i}$, and $C \subseteq \mathcal{R}_{-}$. Now,

$$
0=a u-u a=\sum_{s \leq-1}\left(s \delta\left(u_{0}\right) a_{s}+b_{s}\right) t^{s-1}
$$

where the element $b_{s} \in L$ depends only on $a_{0}, \ldots, a_{s-1}$, and $u$. Equating the coefficients of $t^{s}$ to 0 in the identity above we obtain the infinite system of equations $s \delta\left(u_{0}\right) a_{s}+b_{s}=0$, $s \leq-1$, with unknowns $a_{s}$. For a given $a_{0}$, the system has a unique solution since $s \delta\left(u_{0}\right) \neq 0\left(u_{0} \notin K=\operatorname{ker} \delta\right.$, $\left.\operatorname{char} K=0\right)$ and $L$ is a field. So, the map $C \rightarrow L$, $c \rightarrow c+\mathcal{R}_{-} t^{-1}$, is a $K$-algebra isomorphism, hence $[C(u): K(u)]=\left[L: K\left(u_{0}\right)\right]$.

3. We can write $u$ as $\alpha t^{n}+\cdots$ for some $0 \neq \alpha \in L$ where $n:=\operatorname{deg} u \neq 0$. Let $0 \neq b \in C(u)$. Then $b=\beta t^{m}+\cdots$ for some $0 \neq \beta \in L$ where $m:=\operatorname{deg} b$. The elements $u$ and $b$ commute, so

$0=[u, b]=\left[\alpha t^{n}+\cdots, \beta t^{m}+\cdots\right]=(n \alpha \delta(\beta)-m \beta \delta(\alpha)) t^{n+m-1}+\cdots=\delta\left(\frac{\beta^{n}}{\alpha^{m}}\right) \frac{\alpha^{m+1}}{\beta^{n-1}} t^{n+m-1}+\cdots$,

and so $\frac{\beta^{n}}{\alpha^{m}} \in \operatorname{ker} \delta=K$. The field $K$ is an algebraically closed field, so, for an element $\beta \in C(u)$ of fixed degree $m$, its leading coefficient $\beta$ is uniquely determined by $m$ up to a scalar factor of $K^{*}$. The set

$$
G:=\{\operatorname{deg} c \mid 0 \neq c \in C(u)\}
$$


is a nonzero subgroup of $\mathbb{Z}$ that contains $\mathbb{Z} n$, hence $G=\mathbb{Z} l$ where $l$ is the least positive element of $G$ (and so $l \mid n$ ). Fix an element $v \in C(u)$ with deg $v=l$. We have proved above that, for a given $\operatorname{deg} c$, the leading term of $c \in C(u)$ is unique (up to a factor of $K^{*}$ ). Then, by the choice of $v$, we can choose $\lambda_{1} \in K^{*}$ such that the elements $\lambda_{1} v^{i_{1}}$ and $b$ have the same leading term where $i_{1}:=n l^{-1}$. Hence the element $b_{1}:=b-\lambda_{1} v^{i_{1}}$ has degree $\operatorname{deg} b_{1}<\operatorname{deg} b$. Repeating the same argument for the element $b_{1} \in C(u)$, we can find $\lambda_{2} \in K$ and $i_{2} \in \mathbb{Z}$ such that the element $b_{2}:=b_{1}-\lambda_{2} v^{i_{2}} \in C(u)$ has degree deg $b_{2}<\operatorname{deg} b_{1}$. Continuing in this way, we can find infinitely many elements of $C(u)$,

$$
b_{\nu}:=b_{\nu-1}-\lambda_{\nu} v^{i_{\nu}}, \lambda_{\nu} \in K, \nu \geq 1
$$

such that $\operatorname{deg} b_{1}>\operatorname{deg} b_{2}>\cdots$ and $i_{1}>i_{2}>\cdots$. So,

$$
b=\sum_{\nu \geq 1} \lambda_{\nu} v^{i_{\nu}} \in K\left[\left[v, v^{-1}\right]\right]
$$

thus $C(u) \subseteq K\left[\left[v, v^{-1}\right]\right]$. The reverse inclusion is evident since the elements $u$ and $v$ commute, thus $C(u)=K\left[\left[v, v^{-1}\right]\right]$. A degree argument shows that $\left[C(u): K\left[\left[u, u^{-1}\right]\right]\right]=$ $\left|\frac{\operatorname{deg} u}{\operatorname{deg} v}\right|<\infty$.

Corollary 7.2 Let $\mathcal{D}(X)$ be the ring of differential operators on a smooth irreducible algebraic curve $X$, and let $\Gamma(X)$ be its quotient division ring. Then $\mathcal{D}(X)$ and $\Gamma(X)$ satisfy the commutative centralizer condition.

Proof. By (12), the division ring $\Gamma(X)$ is the quotient ring for the algebra $Q(X)[t ; \delta]$ which is a subring of the formal pseudo-differential ring $Q(X)\left(\left(t^{-1} ; \delta\right)\right)$. The latter is a division ring. By the universality of localization, the division ring of $Q(X)[t ; \delta]$, that is $\Gamma(X)$, is a subring of $Q(X)\left(\left(t^{-1} ; \delta\right)\right)$. So, $\mathcal{D}(X) \subseteq \Gamma(X) \subseteq Q(X)\left(\left(t^{-1} ; \delta\right)\right)$ and $\operatorname{ker} \delta=K$ in $Q(X)$. By Lemma 7.1, the formal pseudo-differential operator ring $Q(X)\left(\left(t^{-1} ; \delta\right)\right)$ satisfies the commutative centralizer condition, hence $\mathcal{D}(X)$ and $\Gamma(X)$ satisfy the commutative centralizer condition.

Corollary 7.3 The ring of differential operators $\mathcal{D}(X)$ on a smooth irreducible algebraic curve $X$, or any non-commutative subalgebra $A$ of the division ring $\Gamma(X)$ with $\mathrm{GK}(A)<3$ admits the Dixmier partition and satisfies both the homogeneous centralizer condition and the commutative centralizer condition.

Proof. Since $K=\bar{K}, A$ is a noncommutative domain, and $\operatorname{GK}(A)<3$, we see that the hypothesis of Corollary 3.2 holds, so $A$ admits the Dixmier partition. The algebra $\Gamma(X)$ satisfies the commutative centralizer condition, so, by Theorem 1.3, the algebra $A$ satisfies both the homogeneous centralizer condition and the commutative centralizer condition.

Corollary 7.4 (An Analog of Dixmier's Problem 6 for $\mathcal{D}(X)$ ) Let $\mathcal{D}(X)$ be the ring of differential operators on a smooth irreducible algebraic curve $X$, and let $f(t) \in K[t]$ be an arbitrary polynomial of degree $>1$. 
1. Let $i=1,2,5$. If $a \in \Delta_{i}(\mathcal{D}(X))$ then $f(a) \in \Delta_{i}(\mathcal{D}(X))$.

2. Let $i=3$, 4. If $a \in \Delta_{i}(\mathcal{D}(X))$ then $f(a) \in \Delta_{5}(\mathcal{D}(X))$.

Proof. Note that if $a \in \mathcal{D}(X) \backslash K$ then $f(a) \in \mathcal{D}(X) \backslash K$. For the element $a \in \mathcal{O}(X) \backslash K$ this fact is evident since $K=\bar{K}$, and for $a \notin \mathcal{O}(X)$ this follows from ord $(f(a))=\operatorname{deg}_{t}(f)$. $\operatorname{ord}(a)>0$. Clearly, the non-scalar elements $a$ and $f(a)$ commute, so $C(a)=C(f(a))$ (Corollary [.2. (1)) since $\mathcal{D}(X)$ satisfies the commutative centralizer condition (Corollary 7.2).

For $i=1,2$, the result follows from Proposition 4.5 .

Suppose that $a \in \Delta_{3,4,5}(\mathcal{D}(X))$. By Theorem 1.2, $f(a) \notin \Delta_{3,4}(\mathcal{D}(X))$, and, by Proposition 4.5 $f(a) \notin \Delta_{1,2}(\mathcal{D}(X))$, hence $f(a) \in \Delta_{5}(\mathcal{D}(X))$.

Question 1. Find generators for the group $\operatorname{Aut}_{K}(\mathcal{D}(X))$ of algebra automorphisms.

Question 2. Classify (up to the action of the group $\operatorname{Aut}_{K}(\mathcal{D}(X))$ ) nilpotent and semisimple elements of $\mathcal{D}(X)$.

Question 3. For a semi-simple element $h$ of $\mathcal{D}(X)$, is the set $\operatorname{Ev}(h, \mathcal{D}(X))$ of eigenvalues for the inner derivation ad $h$ of $\mathcal{D}(X)$ equal to $\mathbb{Z} \rho$ for some $\rho \in K$ ?

Question 4. Let $h$ and $a$ be a semi-simple and nilpotent element of the algebra $\mathcal{D}(X)$ respectively. Is the eigenvalue algebra $D(h, \mathcal{D}(X))$ and the nil-algebra $N(a, \mathcal{D}(X))$ finitely generated (Noetherian)?

Question 5. For which $X$, every algebra endomorphism of $\mathcal{D}(X)$ is an algebra automorphism?

\section{ACKNOWLEDGMENT}

The author would like to thank J. Dixmier for comments [12] on his problems from [1], and D. Jordan and T. Lenagan for pointing out on the paper of K. R. Goodearl [14].

\section{References}

[1] S. A. Amitsur, Commutative linear differential operators, Pacific J. Math. 8 (1958), $1-10$.

[2] V. A. Artamonov and P. M. Cohn, The skew field of rational functions on the quantum plane. Algebra, 11. J. Math. Sci. (New York) 93 (1999), no. 6, 824-829.

[3] H. Bass, E. H. Connel and D. Wright, The Jacobian Conjecture: reduction of degree and formal expansion of the inverse, Bull. Amer. Math. Soc. (New Series), 7 (1982), $287-330$.

[4] H. Bass, G. Meisters, Polynomial flows in the plane, Adv. in Math. 55 (1985), no. 2, $173-208$. 
[5] V. V. Bavula, Generalized Weyl algebras and their representations. (Russian) Algebra i Analiz 4 (1992), no. 1, 75-97; translation in St. Petersburg Math. J. 4 (1993), no. 1, 71-92.

[6] V. V. Bavula, A Question of Rentschler and the Problem of Dixmier, Ann. of Math. 154 (2001), no. 3, 683-702.

[7] V. V. Bavula, Dixmier's Problem 5 for the Weyl algebra, (submitted).

[8] W. Borho, P. Gabriel, and R. Rentschler, Primideale in Einhllenden auflsbarer LieAlgebren (Beschreibung durch Bahnenrume). (German) Lecture Notes in Mathematics, Vol. 357. Springer-Verlag, Berlin-New York, 1973.

[9] W. Crawley-Boevey and M. Holland. Noncommutative deformations of Kleinian singularities. Duke Math. J. 92 (1998), no. 3, 605-635.

[10] J. Dixmier, Représentation irréducibles des algébres de Lie resolubles, J. Math. pures et appl. 45 (1966), 1-66.

[11] J. Dixmier, Sur les algèbres de Weyl, Bull. Soc. Math. France 96 (1968), 209-242.

[12] J. Dixmier, a letter.

[13] T. Hodges, Noncommutative deformations of type- $A$ Kleinian singularities. J. Algebra 161 (1993), no. 2, 271-290.

[14] K. R. Goodearl, Centralizers in differential, pseudodifferential, and fractional differential operator rings. Rocky Mountain J. Math. 13 (1983), no. 4, 573-618.

[15] A. Joseph, The Weyl algebra - semisimple and nilpotent elements, Amer. J. Math. 97 (1975), no. 3, 597-615.

[16] G. Krause and T. Lenagan, Growth of algebras and Gelfand-Kirillov dimension. Revised edition. Graduate Studies in Mathematics, 22. American Mathematical Society, Providence, RI, 2000.

[17] E. E. Kirkman and L. W. Small, $q$-analogs of harmonic oscillators and related rings, Preprint, Wake Forest University and University of California, 1992.

[18] M.-P. Malliavin, L'algèbre d'Heisenberg quantique, C. R. Acad. Sci. Paris, Sér. 1, 317 (1993), 1099-1102.

[19] V. Mazorchuk, A note on centralizers in $q$-deformed Heisenberg algebras. AMA Algebra Montp. Announc. (2001), Paper 2, 6 pp.

[20] J. C. McConnell and J. C. Robson, Homomorphisms and extensions of modules over certain differential polynomial rings, J. Algebra 26 (1973), 319-342. 
[21] J. C. McConnell and J. C. Robson, Noncommutative Noetherian rings. With the cooperation of L. W. Small. Revised edition. Graduate Studies in Mathematics, 30. American Mathematical Society, Providence, RI, 2001.

Department of Pure Mathematics

University of Sheffield

Hicks Building

Sheffield S3 7RH

email: v.bavula@sheffield.ac.uk 